\title{
Le stéréotype, un objet modélisé pour quels usages didactiques?
}

Cadre théorique et analyse d'une expérience d'enseignement de l'écriture littéraire à l'école primaire

Which didactic uses is the stereotype modelled for? Theoretical frame and analysis of a teaching experiment of literary writing at the primary school

Jean-Louis Dufays et Bernadette Kervyn

\section{OpenEdition}

Journals

\section{Édition électronique}

URL : http://journals.openedition.org/educationdidactique/728

DOI : $10.4000 /$ educationdidactique.728

ISBN : 978-2-7535-1623-6

ISSN : 2111-4838

Éditeur

Presses universitaires de Rennes

Édition imprimée

Date de publication : 1 mai 2010

Pagination : 53-80

ISBN : 978-2-7535-1118-7

ISSN : 1956-3485

Référence électronique

Jean-Louis Dufays et Bernadette Kervyn, «Le stéréotype, un objet modélisé pour quels usages didactiques? », Éducation et didactique [En ligne], 4-1 | 2010, mis en ligne le 01 avril 2012, consulté le 10 décembre 2020. URL : http://journals.openedition.org/educationdidactique/728 ; DOI : https:// doi.org/10.4000/educationdidactique.728

Ce document a été généré automatiquement le 10 décembre 2020.

Tous droits réservés 


\section{Le stéréotype, un objet modélisé pour quels usages didactiques?}

Cadre théorique et analyse d'une expérience d'enseignement de l'écriture littéraire à l'école primaire

Which didactic uses is the stereotype modelled for? Theoretical frame and analysis of a teaching experiment of literary writing at the primary school

Jean-Louis Dufays et Bernadette Kervyn

1 L'objectif du présent article est de mettre en dialogue deux approches de la stéréotypie afin d'affiner sa constitution en objet didactique. La première approche, de type modélisant, propose une conceptualisation globale de la stéréotypie, intégrant les différentes manifestations qui en relèvent et recouvrant différentes perspectives théoriques. La seconde approche, de type expérimental, prend appui sur une démarche exploratoire et écologique, où les différents acteurs impliqués tentent, dans un contexte précis, de faire du stéréotype un outil didactique. Si ces deux points de vue visent, chacun à leur manière, le développement de savoirs didactiques à propos de la stéréotypie, leur croisement nous semble opportun pour construire et questionner la valeur didactique de la notion, et dès lors son opérationnalisation dans le champ didactique.

\section{Le stéréotype, un objet didactique à circonscrire}

2 Les phénomènes de stéréotypie, quelles que soient les manifestations qu'ils englobent et la manière dont on les nomme (stéréotype, cliché, lieu commun, idée reçue, prototype, convention, doxa, script, norme générique...), ont toujours constitué un objet clé de l'enseignement des langues-cultures et un révélateur de ses enjeux et de ses valeurs.

3 Cela s'explique d'abord par l'ampleur de son extension. La stéréotypie, comme ensemble de phénomènes (Amossy \& Herschberg Pierrot, 1997), affecte en effet tant la pensée que le langage, et peut être associée à quatre niveaux de réalité au moins: (1) le 
niveau des idées, des convictions, des représentations (correspondant à ce que la rhétorique appelle l'inventio), (2) le niveau des structures configurationnelles (ou thématiques) et linéaires (notamment narratives) des discours (correspondant à la dispositio), (3) le niveau des énoncés verbaux (correspondant à l'elocutio) et (4) le niveau des comportements (correspondants à l'actio).

Qui plus est, dans nos travaux précédents, où nous avons développé une approche synthétique et englobante du stéréotype ${ }^{1}$ (Dufays, 1994 et 2000), nous avons dégagé des diverses définitions du phénomène en circulation dans les sciences humaines neuf traits fondamentaux: (1) l'abondance de ses occurrences dans une diversité de contextes (autrement dit sa banalité), (2) la forte solidarité des éléments qui le constituent (autrement dit son caractère semi-figé), (3) le caractère flou et collectif de son origine, (4) sa permanence dans le temps, (5) son inscription dans la mémoire d'une large communauté, (6) son caractère intertextuel, qui est à la fois la source et le résultat de ses nombreuses réitérations, (7) le caractère spontané, "automatique ", "évident ", non interrogé de la majorité de ses usages, (8) la multiplicité des valeurs qu'on peut lui attribuer: un stéréotype peut être jugé pertinent (ou non) sur le plan de la vérité, de l'éthique et de l'esthétique; (9) la réversibilité des jugements dont il fait l'objet: instrument privilégié de la justesse, de la pertinence et de l'élégance à certains moments (généralement quand on l'utilise), il apparaît à d'autres moments (généralement qu'on on le repère chez les autres) comme un repoussoir de toutes les valeurs (Amossy, 1991, p. 35-41). À cela s'ajoute, dans le cas des stéréotypes d'inventio, un caractère schématisant, synthétique, qui fait du stéréotype l'outil privilégié de toute catégorisation, et partant de toute pensée abstraite.

5 La large extension du phénomène fait de lui un lieu de passage obligé de tout projet éducatif et a fortiori de toute entreprise didactique. D'un simple point de vue pratique, en effet, enseigner une discipline revient à transmettre les savoirs et les savoir-faire qui apparaissent comme les plus fondamentaux de celle-ci: par leur caractère standardisé et très fréquent, les stéréotypes apparaissent donc comme les matières premières de l'école, celles dont l'enseignement se justifie avant tous les autres, et que l'on trouve dès lors en première place dans les manuels et les programmes (Dufays, 1993, 1997).

Vérifions-le immédiatement à propos de l'enseignement du français:

1. les stéréotypes d'inventio sont ces "vérités ", ces « valeurs » et ces " savoirs » premiers et schématiques qu'il convient de transmettre à propos de la langue (son histoire, ses registres, sa valeur mais aussi des courants littéraires (définitions standard du classicisme, du romantisme, etc.)), des genres littéraires (caractérisation du roman, de la poésie, du théâtre, etc.), des notions littéraires (la littérature, la lecture, le personnage, le narrateur, etc.), des événements de l'Histoire littéraire (Querelle des Anciens et des Modernes, bataille d'Hernani, procès intentés à Baudelaire et à Flaubert, révolutions célinienne et kafkaïenne, émergence du Nouveau Roman, etc.), mais aussi des auteurs (traits descriptifs et faits biographiques majeurs de Hugo, de Rimbaud, de Michaux, etc.) ;

2. les stéréotypes de dispositio sont les règles et les conventions propres aux différents genres discursifs et littéraires;

3. les stéréotypes d'elocutio sont d'une part les clichés de langue - formules toutes faites, figures élégantes - d'autre part les structures verbales qui régissent les expressions correctes; 
4. les stéréotypes d'action sont les comportements attendus dans le cadre des tâches scolaires, et qui touchent à des domaines aussi variés que la ponctualité, l'exactitude, le soin, la précision, la modestie, etc. Dans le cadre du cours de français, ces comportements concernent plus spécifiquement l'usage plus ou moins respectueux, démocratique, exigeant que l'on fait de la langue et des textes.

7 La prégnance de la stéréotypie se vérifie également dans les sciences exactes, où les modèles mathématiques, physiques ou chimiques ou biologiques qui sont enseignés apparaissent comme des connaissances savantes opposées aux connaissances communes, aux idées reçues simplistes.

Instrument clé de tout apprentissage (Recherches, 1989), le stéréotype joue forcément un rôle fondamental dans celui de la lecture comme de l'écriture. Pour le vérifier, nous allons à présent nous attacher à décrire sa place dans ces deux processus.

\section{Les usages des stéréotypes dans la lecture}

9 Une partie de nos recherches précédentes a concerné les usages des stéréotypes dans la lecture, et singulièrement dans la lecture littéraire (Dufays, 1991, 1994, 2001; Dufays, Gemenne et Ledur, 2005). Observant la diversité des fonctionnements effectifs de ces phénomènes, nous avons pu montrer qu'ils exerçaient un rôle décisif dans chaque phase du processus de la lecture, et ce quels que soient les compétences et l'âge du lecteur ${ }^{2}$.

10 Avant la lecture, les stéréotypes d'inventio et dispositio constituent une part essentielle de l'horizon d'attente - c'est-à-dire des préconnaissances relatives aux genres, à l'histoire littéraire et aux intertextes - par rapport auquel le lecteur situe le texte qu'il s'apprête à lire. À cet égard, ils aident aussi bien à " précadrer » chaque texte dans une diversité de catégories (en permettant par exemple d'estimer que tel texte est un roman du courant réaliste et qu'il participe d'une conception balzacienne du récit) qu'à les "préjuger» en les associant à des valeurs qu'ils sont supposés illustrer. Ces préconnaissances sont enfin à la base tant du choix des textes à lire que des motivations qui seront mobilisées pendant la lecture - puisque nous ne lisons que les textes dont nous pensons, sur la base des informations dont nous disposons, qu'ils répondront à telles ou telles de nos attentes. Autrement dit, avant même de commencer à lire, nous investissons d'emblée le texte de nos stéréotypes.

11 Cela étant, c'est bien entendu pendant la lecture proprement dite que les stéréotypes exercent leur rôle le plus actif. Dès la perception visuelle, l'identification des mots n'est possible que par la reconnaissance d'associations de lettres et de mots déjà maîtrisés, c'est-à-dire d'images verbales stéréotypées ${ }^{3}$. De même, ces images verbales ne sont sémiotisées, c'est-à-dire dotées de sens, que sur la base d'équivalences routinisées entre certains signifiants et certains signifiés, des équivalences dont l'abondance et l'automatisation progressive définissent en partie la maîtrise d'une langue: connaître le français, c'est entre autres être capable d'associer de manière immédiate des sens à des mots et à des phrases formulés dans cette langue; c'est donc maîtriser les stéréotypes les sens prototypiques - liés à chaque mot. À ces stéréotypes paradigmatiques qui fondent le sens des mots s'ajoutent aussitôt les stéréotypes syntagmatiques, c'est-à-dire les associations standardisées de mots qui permettent de donner sens aux unités du niveau du syntagme et de la phrase. Comprendre une phrase, c'est toujours en partie la 
reconnaître: non pas parce qu'on l'aurait déjà lue telle quelle, mais parce qu'on peut la rattacher à un schéma syntagmatique familier - autrement dit à un stéréotype syntaxique -, ainsi qu'à des associations de mots conventionnelles. Par définition, l'attribution d'un stéréotype - qu'il soit lexical ou syntaxique - à une unité verbale est largement automatique, et c'est cela qui rend possible la rapidité du décodage et l'illusion d'une transparence du langage.

12 L'automatisme n'est cependant jamais assuré: à tout moment, le lecteur peut achopper sur des mots ou des combinaisons de mots qu'il n'arrive pas à intégrer directement dans un schéma: cela peut être dû soit aux limites de sa compétence (il ne connaît pas suffisamment la langue concernée - mais qui connaît jamais parfaitement une langue?), soit à des éléments d'ordre contextuel (la fatigue, l'absence de lumière ou de calme environnant...), soit encore à des spécificités du texte, qui est parfois construit de manière à piéger ou à dérouter son lecteur. Qui plus est, les associations, même quand elles ont fonctionné de manière automatisée, peuvent se révéler inadéquates, c'est-àdire en décalage avec le sens visé par l'énonciateur et/ou perçu par les autres récepteurs. Ceci amène à souligner que l'association d'une unité verbale à un stéréotype constitue en réalité toujours un acte hypothétique, une interprétation, dont la validité n'est jamais totalement garantie a priori. Pour rendre compte de ce fonctionnement, Eco (1985a), à la suite de Van Dijk (1977), a appelé topics les schémas sémantiques hypothétiques - c'est-à-dire les stéréotypes provisoires - que tout lecteur mobilise tout au long de sa lecture, et qui peuvent affecter tous les niveaux de sens, depuis les mots et les phrases (lieu des microtopics) jusqu'au sens du texte dans son ensemble (lieu du macrotopic).

13 À mesure qu'il construit du sens, le lecteur est inévitablement confronté à des indéterminations (Iser, 1985), qui peuvent se manifester sous des formes diverses blancs, résidus, ambiguïtés, contradictions (Dufays, 1994, p.156-157) - et qui empêchent de " clicher » le texte, c'est-à-dire de l'intégrer totalement dans un schéma unificateur. Le lecteur a dès lors le choix: soit il colmate les blancs en recourant à différentes hypothèses plus ou moins vraisemblables, et ce faisant il réduit le texte aux schémas - aux stéréotypes - qu'il a pu mobiliser, soit il opte pour une lecture suspensive qui consiste à maintenir les indéterminations dans leur incertitude. Le premier choix prévaut généralement dans les lectures "fonctionnelles ", dont la visée est de faire signifier les textes de la manière la plus univoque possible - même si Iser considérait le colmatage des blancs comme un acte créatif typique d'une posture esthétique -; le second choix caractérise davantage les lectures «littéraires", qui cherchent au contraire à maximiser les potentialités signifiantes et se montrent réceptives à la polysémie.

14 Enfin, au-delà de la construction du sens « de base ", dont nous venons de décrire le fonctionnement, les stéréotypes jouent encore un rôle fondamental dans les constructions secondes que constituent les différentes formes d'interprétations proprement dites (ou interprétations de niveau 2, si on admet que la construction première est déjà un travail d'ordre interprétatif ${ }^{4}$ ). Interpréter un texte, c'est en effet associer au sens déjà construit un schéma sémantique second, qui se fonde non plus sur ses contenus intrinsèques mais sur des connaissances extérieures que l'on possède à propos de la biographie, de la psychologie ou des autres productions de l'auteur, ou bien de textes écrits par d'autres, ou bien de l'Histoire, grande ou petite, littéraire ou extralittéraire, ou bien du contexte social, culturel, économique ou institutionnel qui 
entoure la production du texte, ou encore d'un système explicatif à visée générale relevant de la psychanalyse, de l'anthropologie, de la religion ou de quelque symbolisme que ce soit. Et il est assez clair qu'un grand nombre des schémas interprétatifs de ce type sont par définition des stéréotypes d'inventio (exemples: le schéma œdipien, le schéma christique, le schéma marxiste, le schéma tragique, le schéma romantique, toutes les formes de schémas mythiques...) susceptibles de se greffer à une infinité de significations premières.

, ce n'est pas seulement comprendre et interpréter. La construction du sens va toujours de pair avec une modalisation du texte, un positionnement psychologique que le lecteur adopte à son égard. On distingue ici deux grandes possibilités.

La modalisation la plus courante est la participation, qui consiste à privilégier la dimension référentielle du texte, c'est-à-dire à lui faire produire une illusion de réalité maximale. Dans ce cas, le lecteur laisse libre cours à ses émotions, à ses sympathies et ses antipathies pour les personnages, à ses peurs, à ses tristesses et à ses joies à l'égard des situations qu'ils traversent. De ce fait, il tend à se projeter dans les situations qu'il lit ou à l'inverse à rapprocher celles-ci de sa propre vie, et s'identifie volontiers à certains personnages, en communiant aux diverses formes de stéréotypies - verbales, thématico-narratives, idéologiques - qui leurs sont associées. Participer au texte revient donc, largement, à adhérer à ses stéréotypies.

Dans le contexte des lectures scolaires, scientifiques et académiques, c'est une tout autre modalité qui prévaut: celle de la distanciation, qui consiste, au contraire, à suspendre la dimension référentielle au bénéfice des observations d'ordre formel, linguistique, stylistiques, rhétoriques ou compositionnels, ou encore à lire le texte comme le symptôme ou l'exemple d'une stéréotypie préexistante. Les différentes formes d'interprétation dont il a été question ci-dessus sont forcément les outils premiers de cette distanciation, qui constitue par nature un acte de méfiance à l'égard de toute forme de stéréotypie.

18 Aucune de ces modalisations n'est spécifique à la lecture littéraire; en revanche, en nous inspirant des travaux de Michel Picard (1986), nous avons défendu (Dufays, 1994, p. 212-215) l'idée que lire littérairement revenait à alterner au maximum des postures contradictoires, à osciller entre ce qu'à la suite de Winnicott, Picard appelle le playing (la participation) et le game (la distanciation), ou encore le lu et le lectant. Se manifeste ainsi une troisième modalité de lecture, celle du va-et-vient concerté (que l'on distinguera du va-et-vient ordinaire, inhérent peu ou prou à toute lecture) entre participation et distanciation et entre les valeurs opposées liées à ces deux modalités. d'évaluer le texte. Et ici encore, c'est sur les stéréotypes qu'il est forcé de s'appuyer en premier lieu. Évaluer un objet, c'est en effet toujours le référer à des standards préétablis, autrement dit à des clichés qui sont censés incarner les modèles ou les prototypes des valeurs attendues.

20 Les critères de valeurs mobilisables pour évaluer un texte sont très divers. Nous en avons retenu au moins sept (Dufays \& Vanhulle, 2002, p. 25): la densité sémantique, la portée référentielle, le rapport aux conventions du genre et du courant, l'esthétique formelle, la portée éthique, la portée psychoaffective et le type de fonction langagière privilégié. 
21 Parmi ces critères, chaque lecteur en privilégie un ou plusieurs - choix qui se fait généralement avant même de commencer à lire -, mais il lui reste ensuite à affecter une valeur positive ou négative au texte au regard des critères choisis. Cette part proprement judicative de l'évaluation est en partie propre à chaque lecteur, mais elle se fait aussi en grande partie sur la base de systèmes de valeurs collectifs. En l'occurrence, les préférences des lecteurs peuvent être référées au moins à deux grandes esthétiques: l'esthétique "classique», qui valorise l'unité (ou la clarté, la simplicité), le souci de réalisme ou de vraisemblance, la conformité aux codes conventionnels, l'harmonie des formes, la conformité à des normes morales dominantes, l'émotion ainsi que la primauté de la fonction référentielle (ou du « fond »), et l'esthétique "moderne », qui valorise à l'inverse la pluralité l'énigme, la complexité, l'indifférence au réalisme, l'originalité (ou la subversion), la dissonance (ou l'éclatement), la transgression des codes moraux, l'impassibilité et la primauté de la fonction poétique (ou de la « forme »).

Entre ces deux tendances, on peut, à la suite d'Eco (1985 b), qualifier de " postmoderne » l'esthétique qui consiste à alterner sciemment entre les deux systèmes de valeurs opposés, c'est-à-dire à apprécier un texte pour des raisons apparemment contradictoires:

On perçoit ici la proximité qui existe entre l'attitude esthétique postmoderne et la lecture littéraire telle que Picard la définit: dans les deux cas, il s'agit de combiner des postures a priori contradictoires. Et cela nous permet de conclure cette section en notant que les lectures postmoderne et littéraire ainsi conçues ne sont rien d'autre qu'une activation concertée de la double face - celle du signe " ordinaire ", " dénoté », « fonctionnel » et celle du signe "marqué », « intertextuel », " problématique »- des stéréotypes.

\section{Les usages des stéréotypes dans l'écriture}

24 Si l'on passe ensuite du point de vue de la réception à celui de la production et qu'on s'intéresse aux usages du stéréotype dans le processus d'écriture, on peut à nouveau distinguer trois cas de figure fondamentaux. En tant qu'outils d'écriture, les stéréotypes peuvent être énoncés comme des signes ordinaires assumés par l'énonciateur (on dira alors qu'ils sont énoncés au premier degré), ils peuvent au contraire être mis à distance et traités comme des citations (on parlera alors de deuxième degré), et ils peuvent enfin faire l'objet d'un traitement oscillatoire (qu'on appellera une énonciation au troisième degré).

L'usage le plus fréquent est sans nul doute l'énonciation au premier degré. Lorsqu'il est utilisé comme un signe ordinaire, le stéréotype exerce, en raison de sa grande fréquence, une série de fonctions extrêmement précieuses pour celui qui écrit: c'est un activateur de la perception (un texte truffé de stéréotypes s'écrit et se lit plus facilement et plus rapidement qu'un texte qui vise à surprendre), c'est souvent un indicateur générique efficace (chaque genre discursif est mobilisé avant tout à travers les stéréotypes qui le caractérisent), c'est un facteur de vraisemblance (il est plus facile de (faire) croire à la réalité d'une description lorsqu'elle est familière que lorsqu'elle est surprenante), c'est un support d'identification et d'émotion (dans bien des cas, on émeut davantage autrui et on suscite davantage sa sympathie quand on lui présente des images, des scènes, des personnages ou des discours qui lui sont familiers que lorsqu'on 
le déroute), c'est un trait argumentatif (la rhétorique antique avait déjà identifié les "lieux communs", les vérités et les types de raisonnements admis par le plus grand nombre comme des outils de conviction très puissants), et c'est aussi, bien souvent, un signal de littérarité (la seule présence de certaines métaphores ou de certaines figures stéréotypées - les roses de la vie, le blanc manteau de la neige -, donne d'emblée à un discours un cachet de poéticité).

Lorsqu'à l'inverse, les stéréotypes sont énoncés au deuxième degré, ils deviennent des signes intertextuels explicites soumis au jugement critique, positif ou non, de l'énonciateur. On distinguera ici le cas des simples citations (" comme le dit le proverbe, il n'y a pas de fumée sans feu»), dans lesquelles l'auteur les assortit de signes métalinguistiques qui montrent clairement qu'il les emprunte à une source existante, le cas des récupérations, où, sans subir d'altération, les stéréotypes sont mis dans la bouche d'un personnage par rapport auquel l'auteur prend ses distances, le cas des transformations, dans lesquels ils sont utilisés sans guillemets ni commentaires mais soumis à des altérations de forme ou de contenu, et le cas des décontextualisations (au sein de sottisiers ou de répertoires, par exemple) dans lesquels ils deviennent l'objet même du discours, sont présentés comme des curiosités, des pièces soumises à la réflexion du lecteur.

Enfin, il arrive, notamment (mais pas seulement) dans certains textes littéraires, que les stéréotypes soient énoncés au troisième degré, c'est-à-dire d'une manière indifférenciée, qui ne permet pas de savoir si l'auteur les assume ou non, partage leurs valeurs ou contraire les désavoue. Pour produire un tel effet d'indifférenciation, l'auteur a le choix entre plusieurs procédés: il peut simplement confronter dans un même texte des stéréotypes opposés - ou des points de vue opposés sur un même stéréotype - en s'abstenant de trancher; il peut aussi énoncer ou mettre en scène des stéréotypes d'une manière ambiguë, en laissant planer le doute sur leur origine ou leur validité. Il est sûr en tout cas que cette manière de mettre en scène les stéréotypes est particulièrement prisée par certains écrivains modernes et postmodernes - comme Flaubert ou Albert Cohen -, mais aussi par des scripteurs relevant d'autres champs professionnels (journalistes, essayistes, publicitaires...) et apparaît comme le fondement même de leur regard sur le monde.

Voilà, résumé de manière très rapide, notre modélisation des usages des stéréotypes dans la lecture et dans l'écriture. Au départ de cela, la question qui se pose aux chercheurs en didactique que nous sommes estla suivante: compte tenu du rôle pluriel et fondamental exercé par les stéréotypes dans tous les domaines de la pensée et de la communication, comment former des enseignants d'une part, et des élèves d'autre part, aux enjeux et aux modalités d'énonciation et de réception de ces phénomènes? Pour rencontrer cette visée praxéologique et épistémologique sans tomber dans une recherche de terrain applicationniste, nous avons entrepris, via une recherche-action, d'analyser comment des acteurs scolaires reconstruisaient le sens et les valeurs du stéréotype en situation d'enseignement et d'apprentissage. C'est cette recherche (Kervyn, 2008 a), centrée sur l'écriture, que nous allons maintenant présenter. 


\section{Le stéréotype comme outil d'enseignement et d'apprentissage de l'écriture: une opérationnalisation en fin d'école primaire}

\section{Démarche méthodologique retenue}

Afin d'investiguer les intérêts de la notion de stéréotypie et des phénomènes qui en relèvent pour l'enseignement et l'apprentissage de l'écriture, nous avons entrepris d'observer des pratiques de classe qui donnent à voir leur mise en œuvre. L'objet de notre recherche (Kervyn, 2008 a) a donc été d'analyser comment un travail scolaire centré sur la stéréotypie peut occasionner, en tant qu'outil, une transformation des pratiques professionnelles des enseignants et des pratiques scripturales des élèves. Il s'agit là d'une analyse de travail en situation, menée dans une perspective résolument écologique.

Pour rendre possible les conditions d'une telle analyse, nous avons suscité un processus d'opérationnalisation de la notion dans deux classes françaises de cours moyen $2{ }^{6}$ en invitant chacun des deux enseignants à reconfigurer le savoir principalement théorique (mis en discussion au sein du groupe de travail ${ }^{7}$ durant un temps de formation initiale ${ }^{8}$ ) en un savoir stratégique pour l'action d'enseignement et d'apprentissage dans le contexte qui était le leur.

Plutôt que de chercher à établir des preuves de l'efficacité d'un tel travail, nous avons privilégié l'observation du processus d'outillage et du processus de construction de significations par les enseignants et les élèves. Cette orientation épistémologique nous a menés vers un dispositif de recherche exploratoire, relevant à la fois de la rechercheaction ${ }^{9}$, de la recherche qualitative (Paquay, Crahay \& De Ketele, 2006), de la recherche collaborative et potentiellement de la recherche innovation.

En l'occurrence, notre analyse porte sur la manière dont deux enseignants expérimentés ${ }^{10}$, préalablement initiés à la problématique de la stéréotypie, font de celle-ci un outil d'enseignement et d'apprentissage de l'écriture pour leurs classes respectives. Nos axes d'analyse privilégiés sont le sens, les fonctions et les valeurs que ces acteurs confèrent à la notion de stéréotypie, en lien avec les contraintes du milieu, les objectifs d'apprentissage scriptural fixés par ces enseignants, leurs styles pédagogiques et la réception par les élèves des traitements de la notion et des phénomènes de stéréotypie proposés par leur enseignant.

33 Nos données sont composées de deux séquences de classe retranscrites, des documents de travail des enseignants, des productions écrites successives des élèves, de leurs réponses à des questionnaires initiaux et finaux, ainsi que de la séance de bilan retranscrite. Tout cela nous a permis d'observer les activités et les discours, oraux et écrits, au travers desquels les enseignants et les élèves ont circonscrit, interprété et investi l'objet et lui ont conféré une pertinence dans le contexte qui était le leur. Notre démarche a dès lors consisté à analyser un processus d'outillage de l'écriture en situation scolaire fondé sur les phénomènes et la notion de stéréotypie, que nous avons considérés dans une perspective avant tout discursive.

34 Cette étude est de nature didactique car elle se situe au cœur du processus d'enseignement-apprentissage et se fonde sur une approche systémique des trois pôles du triangle didactique. En effet, dans notre travail, le pôle enseignant occupe une place 
privilégiée, compte tenu de son rôle dans le processus d'opérationnalisation, mais la prise en compte des élèves nous a paru tout aussi essentielle pour analyser la valeur d'apprentissage de l'outil didactique considéré. Enfin, notre analyse de l'objet scriptural est double puisqu'elle porte à la fois sur sa dimension stéréotypée et sur le genre scolaire dont il relève, en l'occurrence la poésie, qui a été choisie par les enseignants pour réaliser l'opérationnalisation. Ce genre littéraire scolaire occupe une place centrale dans notre analyse car nous pensons qu'il oriente fortement la problématisation, la pertinence et l'usage même des phénomènes et de la notion de stéréotypie.

Notre perspective n'est donc pas celle d'une formulation de prescriptions ou de propositions non expérimentées sur le terrain scolaire. Il ne s'agit pas non plus d'analyser des productions d'élèves sorties de leur contexte de réalisation, à partir d'une modélisation préétablie de la stéréotypie. Enfin, notre perspective n'est pas non plus « purement » ou « directement » praxéologique, puisqu'il s'agit pour nous d'abord de décrire des pratiques pour les comprendre et développer des savoirs didactiques. Ce n'est qu'ensuite que nous pourrions éventuellement envisager une plus vaste transformation des pratiques, mais dans le cadre d'un mouvement bidirectionnel, qui va de la recherche vers la pratique scolaire et vice-versa. Cette conception de la recherche, qui affirme la complémentarité essentielle des apports de la théorie et de la pratique, requiert de garder constamment un équilibre entre les enjeux praxéologiques et les exigences de scientificité. Cet équilibre nous semble capital pour outiller la didactique de l'écriture et ses acteurs sur un plan tant praxéologique qu'épistémologique.

Au final, la démarche globale dont relève notre recherche est celle d'une transposition didactique (Chevallard, 1994; Petitjean, 1998) puisqu'il est question d'exploiter des savoirs "savants" pour mettre en place des pratiques d'enseignement et d'apprentissage, mais cette démarche se fait par et avec la pratique, ce qui favorise la construction de savoirs didactiques, notamment pragmatiques.

\section{Quelle approche de la stéréotypie dans l'écriture?}

Concernant l'écriture et le sujet scripteur, de nombreux travaux, situés dans des champs disciplinaires variés (linguistique, sociologie, psychologie, littérature, didactique...) sont aujourd'hui disponibles. Sans nous étendre sur l'ensemble de ces investigations, nous voudrions mettre en avant deux aspects de l'écriture et du sujet scripteur qui sous-tendent, dans la présente expérimentation, notre approche didactique du stéréotype.

1. Dans la mesure où l'écriture est un processus qui combine une diversité de codes et de modalités de mise en mots qui la rendent hétérogène (François, 2006), les différentes formes de stéréotypie qui la fondent doivent être considérées tant sous l'angle de la stabilité que sous l'angle de la variation. Autrement dit, la stabilité et le figement des stéréotypes ne doivent pas masquer l'hétérogénéité de leurs usages: celle-ci a partie liée avec celle des sujets scripteurs (Chiss, 1993) et des groupes sociaux, mais aussi avec l'hétérogénéité constitutive de la langue en discours, elle-même renforcée par la multiplicité des fonctions attribuées aux pratiques scripturales (moyen de communication, fonction mémorielle, visée esthétique, fonction réflexive...). Ainsi, le stéréotype, en tant que phénomène de reprise intrinsèquement dialogique ${ }^{11}$ (Kervyn, 2008 c), se situe à l'articulation du social, du collectif et de l'individuel: stable et partagé sans être pour autant totalement figé, il constitue une 
entité cognitivo-verbale que les discours véhiculent, investissent et refaçonnent diversement. recherche en didactique demande dès lors de porter attention à toutes ces dimensions ou bien de privilégier l'une d'elles: soit le produit semi-figé, soit son usage singulier à travers une diversité de réalisations (qui vont du renforcement du figement conventionnel jusqu'à son effacement), soit encore le processus permettant de les construire ou de passer de l'un à l'autre. Dans la mesure où nous privilégions l'étude du processus scriptural (Kervyn, 2009 a), notre analyse s'inscrit dans les travaux qui se focalisent sur la reconstruction en discours de la stéréotypie (notamment Galatanu, 2007; Pépin, 2007; Dreyfus, 2007).

1. Nous considérons qu'enseigner l'écriture littéraire à l'école primaire - puisque c'est de cela qu'il va être question -, requiert de faire entrer tous les élèves peu à peu dans une posture d'« écriture littéraire secondarisée » (Bakhtine, 1984), c'est-à-dire dans la mise en œuvre d'une conception de l'écriture relevant davantage de la «sphère littéraire-artistique » que de la «sphère quotidienne ${ }^{12}$ » - ce qui n'implique ni hiérarchisation ni séparation stricte entre ces deux sphères. Pour amener les élèves à développer ce rapport esthétique à l'écriture, il s'agit à la fois de leur faire acquérir des conventions et des stéréotypes et de les sensibiliser à leurs variations, notamment à leurs émergences insolites, sur un plan formel ou thématique, créatrices d'effets de surprise et de curiosité, ainsi que d'une forme de plaisir. Un tel travail nécessite d'instaurer une distance avec le traitement quotidien et spontané que les élèves font des stéréotypes.

Dans le cas spécifique de la poésie, tous les acteurs ${ }^{13}$ s'accordent sur le fait qu'elle est par excellence un lieu d'équivoque, d'oscillations, d'ouverture à l'expérimentation, de variations sociales et historiques: on comprend dès lors que les phénomènes de stéréotypie non mis à distance (tels que les locutions figées, les clichés de langage ou les représentations prototypiques) y reçoivent a priori une valeur négative ou limitative. Les effets de fixité et de retour du même qui caractérisent la stéréotypie (qu'elle soit d'elocutio, de dispositio ou d'inventio) sont en effet en tension avec les valeurs de métamorphose et d'expérimentation qui semblent constitutifs du fait poétique. Sous l'angle didactique, il s'agit alors de voir quels traitements du stéréotype peuvent participer aux valeurs de la poésie.

\section{Inscription dans une visée d'outillage}

L'étude de cas dont nous rendons compte (Kervyn, 2008 a) avait pour visée, nous l'avons dit, d'explorer des possibilités qu'offrent les stéréotypes et la notion de stéréotype comme outils d'enseignement et d'apprentissage de l'écriture poétique en fin d'école primaire. D'un point de vue théorique, au vu des caractéristiques de la stéréotypie (notamment sa prégnance, sa stabilité, sa fréquence et son ancrage collectif), nous avions fait l'hypothèse qu'il s'agissait d'un phénomène et d'une notion susceptibles d'outiller puissamment la didactique de l'écriture.

Dans la perspective historico-culturelle à laquelle nous nous référons pour construire la notion d'outil et d'outillage didactique ${ }^{14}$, le développement passe par un processus d'outillage, c'est-à-dire par l'appropriation d'artefacts - ou plutôt d'usages d'artefacts chargés de culture. Ainsi, l'élève apprend à écrire et développe des compétences scripturales en s'appropriant des artefacts d'écriture qui outillent ses pratiques, tandis

Éducation et didactique, 4-1 | 2010 
que l'enseignant développe des compétences d'enseignement de l'écriture et s'outille en s'appropriant des artefacts relatifs à cette tâche. En d'autres termes, pour autant que les stéréotypes (en tant que phénomènes) et la notion de stéréotype deviennent un moyen d'action en situation (Rabardel, 1999/2002, p. 269), leur utilisation comme outils d'apprentissage ou d'enseignement permet, sur un temps plus ou moins long, le développement de compétences scripturales pour les élèves et de compétences d'enseignement pour les enseignants.

Au-delà de ce point de vue global, nous voudrions mettre en avant six points relatifs à l'outil issus de travaux de disciplines contributives pour la didactique. Si la considération de ces six aspects liés à l'outil a guidé nos choix méthodologiques et l'orientation épistémologique de notre travail, nous pensons que, outre le poids de l'ancrage disciplinaire, ils sont susceptibles de concerner toute recherche didactique inscrite dans une visée d'outillage.

1. Tout d'abord, qu'on se situe en psychologie ou en didactique, il faut garder à l'esprit l'importance prépondérante qu'occupe le sujet dans le processus d'outillage. En effet, l'artefact - que constitue ici le stéréotype - n'est pas en soi un outil. Il le devient quand il est institué dans l'action par le sujet, qui en fait un moyen pour atteindre le(s) but(s) de son action dans une situation précise (Clot, 1999/2004, p. 124). Il n'est dès lors pertinent que par rapport à une ou des actions personnelles situées, qui par ailleurs l'enrichissent (notamment en lui ajoutant une nouvelle fonction ${ }^{14}$ ) et le caractérisent.

Cette dimension renvoie à un des traits définitionnels énoncés par Rabardel (1997), à savoir le fait que l'outil (ou l'instrument) se situe obligatoirement entre le sujet et l'objet, et qu'il est adapté à l'un et à l'autre. Clot (1999/2004, p. 123), pour sa part, souligne que l'instrument possède "une double vie», "participant à la fois au mouvement des organisations sociales et à celui des dispositions personnelles, à la fois signification sociale et sens personnel ». En cela - et c'est bien le cas pour le stéréotype dans l'écriture -, l'outil est plus que situé entre deux entités, il se développe comme « une entité mixte » (Ibid.).

En termes didactiques, on peut d'ores et déjà se demander comment cette part du sujet dans l'outil va se manifester et/ou être prise en compte. L'enseignant et le didacticien doivent-ils penser l'outil de façon plurielle, de façon éclatée, multifonctionnelle ou «multi-niveaux » pour répondre à la probable hétérogénéité liée à la diversité des sujets apprenants au sein de l'unité classe? Un des défis et des rôles clés pour l'école, dans la configuration sociale qui est la sienne, est bien de permettre l'apprentissage au sein du groupe classe. S'inscrire dans une perspective d'outillage didactique demande alors, selon nous, de déplacer le regard de l'individu vers le contexte classe et ses individus. Cette optique amènerait à considérer qu'il n'y a outillage didactique que si le but atteint ou à atteindre du point de vue du sujet (enseignant ou élève) correspond au but préconisé ou prescrit, ou à sa visée développementale. Cette restriction laisse entendre de possibles tensions ou non-correspondances entre l'outil psychologique et l'outil didactique.

1. Nous voudrions ensuite insister sur le fonctionnement interactionniste social de l'outillage et sur son passage progressif de l'interpsychique à l'intrapsychique, c'est-à-dire le passage de formes d'activité sociale et collective à des fonctions outillantes individuelles par la reprise pour soi des deux versants de l'interaction (Brossard, 1989, p. 55). Son espace mental est monogéré mais il est social par son origine qui lui assure une dimension dialogale intériorisée. Cette modalité d'apprentissage et d'appropriation de la fonction outillante nous est apparue pleinement dans les deux classes que nous avons suivies. En effet, nous avons 
été frappés de voir combien les échanges entre les enseignants et les élèves sur les représentations stéréotypées y étaient progressivement repris, prolongés par des échanges entre élèves et devenaient matière à des questionnements et à des propos singuliers de leur part ${ }^{15}$. En cela, il apparaissait nettement qu'outiller, c'est bien développer l'individuation, entendue comme la capacité à prendre peu à peu en charge les deux versions de l'interaction et une partie (ou tout) de ce qu'ils véhiculent: logique, représentation, valeur, sens, contexte, pratique, forme langagière, etc. Ainsi, lorsque nous cherchons à outiller le scripteur, nous voulons lui permettre d'acquérir une progressive autonomie scripturale via l'intégration et la construction collective d'outils scripturaux. D'un point de vue méthodologique, analyser cette individuation progressive passe, pour partie au moins, par une attention, dans la classe, aux mouvements discursifs, aux reprises et aux transformations au sein des échanges oraux et écrits.

2. D'un point de vue didactique encore, l'outil exerce auprès des enseignants puis des élèves des effets de transformation (Vergnaud, 2000) qui peuvent être de nature et d'appellation variée: complexification, déplacement, élargissement, amplification, substitution, réorganisation, restructuration, reconstruction, recomposition, reconstitution, recréation... Dans certains cas, acquérir un «nouvel» outil revient à complexifier la fonction ou la signification d'un outil déjà maîtrisé, à en préciser l'espace d'usage en le mettant en relation avec d'autres usages ou d'autres situations de pertinence. D'autres fois, le sujet adopte un outil nouveau en le greffant sur un outil existant qui, du coup, voit son utilité réduite ou transformée. D'autres fois encore, l'adoption d'un nouvel outil entraine une reconstruction de toute la chaîne d'actions impliquées dans le comportement. C'est en tout cas à l'ensemble $\mathrm{du}$ processus de transformation et à toutes ses formes possibles que le didacticien doit prêter attention.

3. En situation scolaire, observer le processus de transformation attaché à l'outillage revient à observer un ensemble d'activités ${ }^{16}$ et de choix didactiques plus ou moins explicites. Car, comme le rappelle Rabardel, l'artefact ne devient outil qu'à l'usage. À sa composante artefact, l'outil associe forcément une dimension d'usage, qui peut être davantage propre au sujet ou davantage résulter de l'appropriation de schèmes sociaux transmis plus ou moins formellement au sein des communautés qui l'utilisent. Il apparaît ainsi comme étroitement associé à des opérations de travail ou d'activités, de connaissances, de valeurs, de rapports, de fonctions, d'interactions, voire de situations qui, en usages, influent sur lui (Clot, 1999/2004). D'un point de vue méthodologique, observer le processus d'outillage à l'œuvre dans l'enseignement et l'apprentissage de l'écriture nécessite dès lors bien de porter attention aux usages de l'artefact et aux différentes dimensions qui y sont attachées, ce qui dépasse de loin, par exemple, le simple relevé de stéréotypes présents dans un texte d'élève.

4. Selon les situations et les genres de situation où il est mis en œuvre, un artefact change fréquemment de fonctions. Ainsi, le stéréotype exercera probablement des fonctions différentes selon qu'il sera mis en scène en littérature ou en sciences, à l'école ou dans une situation quotidienne extrascolaire. C'est ce que Rabardel appelle la «valeur fonctionnelle située» ou le «sens instrumental en situation»(1999-2002, p. 275). On peut faire l'hypothèse que l'élève aura à acquérir cette variabilité situationnelle et disciplinaire en apprenant à adapter son usage de l'artefact à la situation et à la discipline scolaire dans laquelle il est impliqué. De même, l'enseignant va être amené à doubler l'usage et le sens courant et partagé de l'outil avec des usages didactiques disciplinaires plus spécifiques. Enseignant et chercheur auront aussi à être attentifs à la manière dont se construisent et se négocient ces valeurs fonctionnelles différemment situées. En quoi, par exemple, le sens et la valeur couramment attribués au terme stéréotype interfèrent avec son usage et sa valeur didactique?

5. En situation d'outillage, on est bien en présence à la fois d'une ouverture des possibilités et de la mise en place d'un système plus contraint. Dans cette optique, pour que la stéréotypie 
devienne un outil didactique, il semble donc nécessaire de relever aussi bien les diverses contraintes qui, dans une discipline et dans des situations identifiées, conditionnent sa valeur d'outil que les possibilités auxquelles il se prête, sur le plan de l'action enseignante comme sur celui des apprentissages.

\section{Présentation succincte du travail réalisé dans les deux classes}

Avant d'entrer dans l'analyse des données sous l'angle de l'outillage didactique, il nous semble nécessaire de présenter succinctement les pratiques de classes effectives ${ }^{17}$ qui composent une part majeure ${ }^{18}$ de l'opérationnalisation, soit deux séquences de chacune huit séances d'une durée de vingt à quatre-vingts minutes. Sans entrer dans le détail des activités menées ${ }^{19}$, contentons-nous de relever les objectifs des deux séquences telles qu'elles se sont passées.

L'enseignant 1 auteur de la première séquence (S1) s'est donné comme objectif global de développer la compétence d'écriture poétique de ses élèves et de les ouvrir à une culture et à une pratique littéraires (plus) diversifiées en favorisant un traitement langagier plus conscient et relativisé ${ }^{20}$ des stéréotypes d'inventio liés à la poésie en tant que genre scolaire ${ }^{21}$. Cet objectif global, lui-même ancré dans l'objectif d'opérationnalisation, se décline en trois objectifs plus précis, qui ont été mis en œuvre en 25 phases ou activités successives, chacune répondant à un ou à plusieurs objectifs ${ }^{22}$. Le premier objectif fixé par l'enseignant consistait à vérifier la présence, chez les élèves, d'une vision stéréotypée de la poésie et d'en même temps recueillir des données qui allaient pouvoir être exploitées dans la suite du dispositif. Le deuxième objectif, qui découlait du premier, consistait à traiter scolairement les stéréotypes relevés, en faisant émerger le phénomène et certains de ses effets, en montrant sa diversité dans la poésie et en construisant la notion de stéréotypie. Le troisième objectif visait à transformer le travail sur les stéréotypes, sur la diversité du fait poétique et sur la notion de stéréotype en outil d'écriture poétique, en insistant sur les traitements possibles du stéréotype.

Le second enseignant a, pour sa part, axé sa séquence (S2) sur la production «imaginative " de poèmes sur le thème de la mer, à destination possible d'élèves d'autres écoles joignables via internet. Pour mener à bien cet objectif, qui intègre ou " raccroche » le projet d'opérationnalisation, l'enseignant 2 a mis en place six séances de vingt à quatre-vingts minutes, au sein desquelles trois objectifs plus précis sont apparus. Le premier consistait à réaliser une écriture « diagnostique ", c'est-à-dire qui donne à voir les représentations sur la mer utilisées dans l'écriture et la maîtrise en production écrite du genre scolaire poésie, et ainsi les possibles stéréotypes présents à ces deux niveaux. Au vu des résultats observés, deux autres objectifs ont été fixés et travaillés. Il s'est agi d'abord de faire émerger le caractère stéréotypé, dans l'écriture, de la représentation de la mer chez les élèves - on est à nouveau ici dans les manifestations langagières d'une stéréotypie d'inventio - pour susciter une écriture véhiculant un autre point de vue sur la mer. D'autre part, l'enseignant a travaillé sur la dimension formelle du genre scolaire poésie par des relevés de ce qui le caractérise ou non en réception et en production, et a ensuite réinjecté ces connaissances dans l'écriture pour que les élèves produisent peu à peu des textes plus clairement identifiables comme textes poétiques. Avant la fin de la mise en œuvre du premier objectif, l'enseignant envisageait le troisième, comme le deuxième, de manière ouverte car, d'un côté, les élèves avaient déjà écrit à plusieurs reprises des textes poétiques en 
soignant la forme. Selon lui, certaines caractéristiques formelles du genre étaient alors connues des élèves, et l'écriture pouvait présenter un aspect générique stéréotypé, comme dans l'autre classe. De l'autre côté, comme il le dit lors du bilan, le travail antérieur relevait davantage de l'écriture créative singulière que du travail d'ancrage explicite dans le genre.

\section{Ce que nous apprend l'analyse des pratiques des enseignants et des élèves}

Étudier la construction de l'outil en nous focalisant sur l'activité enseignante revient en fait à mener une double analyse car il s'agit d'envisager tant le processus d'outillage orchestré par les enseignants pour les élèves - observation du scénario élaboré par chaque enseignant pour l'apprentissage de l'écriture poétique de ses élèves - que le processus d'outillage tel qu'il se manifeste dans la pratique des enseignants eux-mêmes - observation de l'outillage de leur propre pratique professionnelle. Sans entrer dans une analyse complète des données recueillies, nous allons envisager, pour le premier volet, la manière dont la notion de stéréotype est introduite, présentifiée et problématisée auprès des élèves, les rôles, les valeurs, les significations et l'importance qu'elle prend, précisément dans l'activité d'écriture poétique. On se demandera alors en quoi les tâches qui ont été proposées et réalisées favorisent certains usages du stéréotype, les détournent d'autres usages ou d'autres significations, les diluent, les récupèrent, les noient... Dans le second volet, nous poserons la question de la transformation de l'activité enseignante: de quelle nature et de quelle forme est-elle? Est-elle de l'ordre de l'innovation pour les enseignants impliqués? Enfin, nous compléterons ces propos par une analyse des données, notamment textuelles, recueillies auprès des élèves, afin de préciser en quoi le stéréotype et les savoirs sur les stéréotypes participent au développement de compétences scripturales.

\section{Le choix d'un travail sur le stéréotype dans l'écriture poétique}

Comme on le voit dans la présentation des séquences, le premier choix opéré par les deux enseignants expérimentateurs est double: opérationnaliser le stéréotype, parmi les phénomènes de stéréotypie envisagés collectivement ${ }^{23}$, et ancrer l'opérationnalisation du stéréotype dans un travail sur la poésie, en tant que genre littéraire scolaire. Cette focalisation conjointe sur l'écriture poétique scolaire et sur le stéréotype et les savoirs qui le concernent positionne le travail dans un domaine et une problématique spécifique: ceux de la création, de l'invention, de l'imaginaire et de l'imagination, du sens esthétique, de la culture littéraire.

Pour motiver leur choix d'une opérationnalisation du stéréotype dans l'écriture poétique, les enseignants ont invoqué des besoins d'ordre scolaire, relatifs à l'enseignement et à l'apprentissage de la littérature. L'enseignant 1 met en avant une première approche assez frustrante de l'écriture poétique avec cette classe. Avec le procédé de reprise du texte initial "à la manière de...", l'enseignant avait eu l'impression forte d'avoir favorisé une écriture "recette", bien pauvre pour de la poésie. Mais comment, au sein d'une tâche scolaire, aboutir à des écrits plus diversifiés, et faire en sorte que les élèves puissent créer ou être davantage engagés dans l'écriture personnelle et moins dans l'application d'une recette? Par ailleurs, au cours de cette séquence, il avait été frappant pour l'enseignant, sensibilisé aux phénomènes de 
stéréotypie lors de la formation initiale et travaillant sur les conceptions initiales en sciences, de constater que, pour beaucoup d'élèves, la vision de la poésie semblait très stéréotypée, ce qui en permettait et en limitait sans doute la réception et la production; ceci confirme tout l'intérêt de la notion de stéréotype en tant qu'outil d'analyse scripturale.

51 L'enseignant 2, pour sa part, semble avoir été motivé, dans son choix de travailler sur les stéréotypes, par trois facteurs didactiques également. Le premier est de poursuivre et de compléter la découverte de l'écriture poétique déjà réalisée, par le travail de composantes et de dimensions scripturales jusqu'alors peu envisagées et qui permettent d'approfondir un peu plus l'écriture personnelle, créative et l'expérience littéraire de la poésie. L'enseignant souhaitait par ailleurs que le travail sur la stéréotypie et sur l'écriture littéraire prenne place dans le projet pluridisciplinaire de la classe consacré au thème de la mer. On voit aisément comment s'est imposée l'idée d'une écriture poétique à partir d'une exploration de la composante thématique du projet. Dans le rapport avec l'opérationnalisation de la stéréotypie, il a paru intéressant à l'enseignant d'observer comment le « sujet mer » ou le « monde mer » était construit et reconstruit par l'écriture, et d'observer si cette reconstruction empruntait et dégageait une vision stéréotypée du thème de la mer. De même, comme nous l'avons déjà signalé, il comptait observer si les formes poétiques qu'allaient adopter les élèves ne présentaient pas un aspect trop stéréotypé, pouvant, dans un cas comme dans l'autre, peut-être faciliter l'écriture, mais limiter la créativité.

Un troisième motif, et non des moindres, a poussé cet enseignant à privilégier un travail sur les représentations stéréotypées et leurs expressions: sa transversalité dans l'apprentissage ${ }^{24}$. Qu'il s'agisse de la représentation du nombre décimal en mathématiques ou de la représentation d'un mot en arts visuels, la notion de stéréotype peut être réinvestie, chaque fois pour pointer une représentation socialement partagée, fréquente, pas toujours vraie, associant de façon relativement figée deux éléments. Selon la situation, la représentation se manifeste et prend forme au travers d'un raisonnement mathématique oralisé ou dans le dessin réalisé. Ainsi, suite à la formation initiale et avant d'entamer l'opérationnalisation en littérature, l'enseignant s'en était déjà servi, précisément dans les deux cas de figure mentionnés. Dans le premier cas, il avait été sensible à l'importance de repérer et de prendre en compte les stéréotypes en tant que phénomènes partagés pouvant tout à la fois entraver les apprentissages et mettre en avant des connaissances existantes, potentiellement tremplin pour les apprentissages. Dans le second cas, il était question, comme en littérature, de représentations spontanées qui influaient sur les productions artistiques et les rendaient quasiment identiques, témoignant ainsi de l'imaginaire collectif.

Mettre en évidence les motifs de sélection de la poésie et du phénomène de stéréotypie opérationnalisé permet de pointer des façons dont la notion de stéréotype peut être problématisée à l'école et en littérature, ou des façons dont il peut rencontrer les besoins des enseignants en quête d'instruments les aidant à penser et à réaliser leurs enseignements. Ainsi, au travers de ces motifs, ressort une première valeur ou fonction attachée à l'outil stéréotype en littérature: sa notion permet, pour ces enseignants, de penser et de mener différemment l'enseignement de l'écriture poétique. À ce titre, elle outille entre autres les enseignants dans leur lecture évaluative des écrits des élèves. La notion de stéréotype constitue de surcroît, nous l'avons évoqué, un outil 
pluridisciplinaire permettant d'interroger notamment les représentations initiales et leurs expressions.

\section{Sens attribué au terme de stéréotype} caractéristiques évoquées en début d'article. On constate aussi que l'utilisation de la notion de stéréotype correspond assez bien aux définitions du phénomène, proposées lors du temps de formation initiale (T1), à savoir « représentation mentale culturelle préconçue; produit de l'imaginaire social, non fondé sur l'expérience et par lequel nous typifions ou généralisons le réel » et "partie de la signification ou représentation simplifiée, conventionnelle et courante, associée à un mot ».

\section{Types de stéréotypes traités}

Bien que les deux enseignants se soient intéressés au même niveau de stéréotypie, leurs choix divergent quant au type de stéréotype traité, puisque l'un s'est focalisé sur la 
forme poétique ou sur la composante formelle ${ }^{28} \mathrm{du}$ genre scolaire poésie, et l'autre, sur le contenu thématique. Si les deux dimensions ${ }^{29}$ participent à la caractérisation du genre, entendu dans un cadre bakhtinien, et " [...] fusionnent indissolublement dans le tout que constitue l'énoncé [...] » (Bakhtine, 1984, p. 265), le premier pose l'épineuse et difficile question du repérage et de l'identification formelle de la poésie, hors de l'école et à l'école. Il nous semble ainsi que ces deux objets ou composantes scripturales ne présentent pas le même degré ou le même type de complexité dans le cadre de l'écriture poétique. Ceci dit, l'enseignant 2 effectue lui aussi un travail sur la construction formelle du genre, mais plutôt en sens inverse puisque sa démarche initiale de recueil des données avait mis au jour un faible marquage poétique dans les textes produits.

On voit aussi combien ces orientations, différentes quant à l'objet stéréotypé (contenu thématique ou structure formelle), s'inscrivent dans des enjeux littéraires à la fois spécifiques et complémentaires qu'exprime l'objectif général de chaque séquence: développer la part de l'imaginaire dans l'écriture littéraire d'un côté, et favoriser l'entrée dans une culture et dans des pratiques littéraires plus diversifiées de l'autre ${ }^{30}$.

61 Il est clair que la pertinence du rôle outillant du phénomène et de la notion de stéréotype pour l'écriture poétique scolaire est à évaluer au regard des ces enjeux et au regard de leur effet transformant sur les pratiques ou les compétences scripturales des élèves. Néanmoins, on peut déjà souligner que la notion de stéréotype est susceptible d'outiller, du côté de l'enseignant, tant le travail poétique du thème que celui de la forme.

\section{Vers une valeur neutre ou fonctionnelle du stéréotype}

Dans les deux classes, il est question de relever la présence potentielle du stéréotype, de le faire émerger, de le rendre plus conscient, de l'étiqueter langagièrement, pour en minorer l'effet restrictif et tenter d'écrire autrement. Dans les deux classes, le stéréotype relevé en début de séquence reçoit alors une valeur initiale quelque peu péjorative, celle couramment associée d'idée ou de représentation toute faite, "pas toujours vraie ", partagée par un grand nombre, « dépourvue d'originalité (classe 1), dont on peut « sortir " pour faire " varier » l'écriture (classe 2). Sa valeur négative dans le cadre de l'écriture poétique est clairement liée à la non-variation qu'il suscite dans le traitement thématique ou à la définition trop rigide et restrictive qu'il incite à donner de cette forme d'écriture. Autrement dit, dans les deux classes, l'aspect négatif central vient de son effet de schématisation par rapport à l'hétérogénéité du réel: un poème n'a pas toujours de strophes, de vers; la mer n'est pas toujours associée aux vacances.

Les termes négativement connotés associés au stéréotype ou à l'élément stéréotypé relevé sont présents de façon plus nette dans les propos et la séquence de l'enseignant 1. Hors de la classe, il parle de "casser les stéréotypes", de les «mettre à mal ». En classe, ces termes ne sont pas utilisés. Il est alors question du fait que «le stéréotype peut être un frein à l'originalité, à la création ", qu'il peut bloquer l'écriture en faisant du stéréotype une nécessité absolue. Par ailleurs, le stéréotype et le travail sur le stéréotype sont aussi valorisés en tant que soutien possible de l'écriture. L'enseignant 1 verbalise ces deux aspects à de nombreuses reprises en classe et discute avec les élèves de cette fonction d'aide ou de ce risque d'entrave: 

scripturale: "frein" et "aide», processus inhibant l'écriture et ressource pour l'écriture. Néanmoins, dans les deux cas de figure, c'est bien le travail de prise de conscience qui confère au stéréotype une valeur "plus neutre ». C'est sur cette base qu'a lieu l'écriture du second poème: les élèves sont invités à prendre appui sur les stéréotypes vus, soit en en reproduisant soit en s'en distanciant. Pour ce qui est du frein à l'originalité, on peut le lire comme une influence ou une incursion du sens couramment attribué au terme stéréotype: la consultation dans la classe du dictionnaire mentionnant l'acception courante d'une reprise dénuée d'originalité a quelque peu aiguillé l'enseignant ainsi qu'une minorité d'élèves vers une valeur péjorative et en conséquence vers une tentative d'évitement du stéréotype, en tension avec sa valeur fonctionnelle.

Leignant 2 , pour sa part, ne présente pas aux élèves ce phénomène comme aussi négatif ou à éliminer. Dans sa classe, c'est beaucoup plus le mode du constat d'un fonctionnement que celui de la critique que nous relevons. Les élèves constatent la présence de leur stéréotype, à partir de quelques marqueurs et sont invités à en « sortir » par l'imagination d'un autre point de vue sur la mer:

La valeur que ce traitement confère aux stéréotypes est simplement cognitive: ils renvoient à un mode de fonctionnement. D'ailleurs, à la fin de la séquence, lors du bilan, l'enseignant réalise que la réécriture s'est faite également sur la base d'un recours aux stéréotypes, à d'autres stéréotypes sur les pêcheurs, le Sri Lanka, les dauphins, etc.: «[...] et en fait du coup on est passés d'un stéréotype à un autre ».

En conséquence, dans cette classe, on est non seulement en présence d'un élément de connaissance, mais aussi d'un ressort, conscient ou non, pour faire varier l'écriture. La valeur du stéréotype est ainsi nettement fonctionnelle et son traitement en classe n'a dévoilé qu'en partie son fonctionnement ou sa place dans le processus scriptural.

\section{La modalisation didactique du stéréotype: le casser ou le mettre en mouvement?}

Nous voudrions poursuivre en dégageant tant ce qui se dessine comme des contraintes majeures pour optimiser l'outillage que des potentialités de cet outil dans le cadre de l'enseignement et de l'apprentissage de l'écriture poétique.

69 Travailler un écrit aussi varié et transgressif que la poésie rend nécessaire de relativiser les indices de poéticité traités de manière stéréotypée, de les multiplier pour approcher un tant soit peu cette diversité. En termes de traitement des stéréotypes et en ce qui concerne l'opérationnalisation réalisée dans la classe 1 d'abord, ce premier constat valide sa démarche déclarée, qui consiste à " casser » les stéréotypes pour ouvrir à la diversité, tout en la nuançant fortement et fondamentalement. En effet, si le figement cognitif et verbal du stéréotype s'oppose bien au renouvellement et à la diversité poétique, ce qui lui confère une valeur initiale plutôt négative de phénomène limitant l'expérience poétique, son traitement en classe s'apparente bien plus à une mise en mouvement des stéréotypes constatés, plutôt qu'à leur démolition ou leur éviction.

70 La nécessaire recherche d'une mise en mouvement, plus que d'un "cassage » ou que d'une suppression, s'explique d'abord par la nature même du stéréotype. En effet, d'une part, même s'il fige et schématise, ce phénomène n'est pas pour autant dénué de pertinence (les poèmes ont souvent des rimes), d'autre part il (pré)existe socioculturellement en dehors de la communauté classe (où il peut avoir sa légitimité et

Éducation et didactique, 4-1 | 2010 
sa pertinence) et se reforme de façon variable contextuellement. De ce point de vue, les stéréotypes repérés dans la classe en littérature ou dans d'autres disciplines continueront à circuler dans l'espace socioculturel qui leur donne forme, quelles que soient les initiatives prises dans la classe, tout comme, d'ailleurs, les savoirs sur les stéréotypes utilisés ou visés en classe continueront à circuler dans les communautés scientifiques de référence.

71 Néanmoins, le travail effectué transforme les stéréotypes et la notion de stéréotype au sein de la classe et en chaque sujet de la classe, tout comme le travail sur les stéréotypes et sur la notion transforment potentiellement les sujets de la classe, leur rapport à la poésie ou à l'écriture poétique, par exemple. Il n'est dès lors pas abusif de parler de stéréotypes et de savoirs sur les stéréotypes qui, en classe, dans et par l'activité discursive, scripturale, réflexive de tel ou tel élève et de l'enseignant, sont mis en mouvement, se transforment, changent de valeur. On a affaire à des objets sociaux tout à la fois stabilisés et qui cheminent dans la classe en se transformant singulièrement. Dans ce contexte collectif, le traitement dominant du stéréotype consiste à le mettre en mouvement par des opérations diverses - et notamment par l'apport de la notion de stéréotype - en vue de transformer à la fois les stéréotypes, les sujets élèves et leurs savoirs, leurs pratiques, leur rapport à la poésie et à l'écriture poétique. Et cette opération entraîne une transformation de la notion de stéréotype, autrement dit sa scolarisation, entendue comme un ajustement à la sphère scolaire ou à la communauté discursive classe (Bernié, 2002), à ses acteurs et à la discipline littérature.

72 La mise en mouvement se justifie aussi par le processus d'apprentissage et d'outillage où l'acquisition de savoirs plus complexes n'annule pas les savoirs quotidiens, mais les transforme. De plus, l'apprentissage et l'appropriation de la fonction outillante s'effectuent par de nombreux échanges entre les enseignants et les élèves sur les représentations stéréotypées, sur les indices verbaux de cette stéréotypie, échanges progressivement repris, questionnés, intériorisés, prolongés par des élèves qui en tirent des propos singuliers.

73 En situation, ces mises en mouvement cognitivo-verbales, qui transforment diversement les représentations stéréotypées ainsi que leurs manifestations langagières, vont d'une manière ou l'autre atténuer le figement existant, chez les élèves, entre la poésie et les indices stéréotypés. Ces mises en mouvement vont relativiser la prépondérance de ces indices, leur caractère incontournable, notamment en tant qu'arguments d'autorités dans le débat (c'est ou ce n'est pas un poème), sans pour autant les gommer ou nier leur marquage en tant que stéréotypes. Par cette orientation, les représentations stéréotypées et leurs traces langagières sont appelées à perdre peu à peu leur valeur négative au profit d'une valeur plus relative liée à un amoindrissement du processus cognitivo-verbal de stéréotypisation, en situation de travail scolaire sur la poésie.

\section{Une modalisation dominante: la distanciation ${ }^{31}$}

74 Si l'on prend en compte l'ensemble du corpus, il est frappant de constater que la grande majorité des mises en mouvement des stéréotypes repérées s'inscrivent dans un processus de distanciation. Appelé et légitimé par les objectifs littéraires formulés par les enseignants et fixés pour des enfants de fin d'école primaire, ce processus de distanciation correspond aussi à un mode ou à une posture générale attendue à l'école 
(Alcorta, 2009; Daunay, 2002) qui consiste, pour apprendre, à mettre à distance les ressources de leur culture spontanée ou déjà acquise.

Au-delà de ce constat, l'analyse des données a permis de dégager sept opérations qui, bien que non exhaustives, participent à la distanciation par rapport aux stéréotypes (relatifs soit à la forme poétique soit au thème) et, au final, à la mise en place chez les élèves d'une écriture poétique plus secondarisée. Dans leurs mises en œuvre, loin d'être exclusives l'une de l'autre, ces opérations se croisent régulièrement au cours d'une séance, voire d'un même échange et, sans doute, se renforcent mutuellement. Vu leur imbrication, nous préférons parler d'un effet global de distanciation discursive et textuelle constatée en cours et en fin de séquence plutôt que d'un effet spécifique de l'une ou l'autre d'entre elles.

76 Ainsi, dans les classes, le processus de mise à distance s'opère, selon les situations, par le biais d'une ou de plusieurs des démarches suivantes:

1. la relativisation de la portée du stéréotype jusqu'alors perçu comme incontournable («un poème ça a des rimes mais ce n'est pas obligé »);

2. la multiplication des indices ${ }^{32}$ de poéticité ou des points de vue sur le thème ou sur la poésie, pour approcher un tant soit peu la diversité poétique;

3. le déplacement de l'attention vers un autre point de vue (plutôt que vers de multiples « nouveaux » ${ }^{33}$ indices ou points de vue ${ }^{34}$ );

4. le passage du cas particulier à la catégorie ${ }^{35}$, c'est-à-dire l'insertion de l'indice stéréotypé prototypique (Kleiber, 1990/2004) dans un espace de fonctionnement et de ressources créatives plus vaste (replacer les rimes dans la catégorie plus vaste de la sonorité poétique, de l'expressivité sonore);

5. l'introduction du jeu, de la surprise ou de l'étrange par un traitement décalé du stéréotype, nécessaire pour qu'il devienne zone d'étrangeté poétique ${ }^{36}$;

6. la prise de conscience qu'il s'agit de stéréotypes et la prise de conscience de leurs effets via une monstration, par l'enseignant, du phénomène et de ses effets. Cette prise de conscience permet aux élèves de passer d'un usage individuel du stéréotype non conscient à un constat explicite de stéréotypisation partagée et généralisée. Prendre conscience qu'une représentation est stéréotypée permet aussi de comprendre pourquoi on a régulièrement tendance à y revenir et à penser de cette manière ${ }^{37}$;

7. la problématisation et l'intégration d'autres repères culturels et historiques référents pour la discipline pour faire entrer dans la classe et y élargir la dimension institutionnelle.

$77 \mathrm{Au}$ final, ces diverses opérations transforment le rapport que les élèves ont aux stéréotypes traités et les rendent plus opérationnels pour atteindre les objectifs disciplinaires fixés. Autrement dit, en cela, les stéréotypes, par le traitement opéré, prennent une valeur fonctionnelle d'outil pour l'enseignement-apprentissage de la poésie et de l'écriture poétique.

Si l'on s'interroge sur la portée des opérations pointées, on remarque que d'autres disciplines soulignent leur importance. Ainsi, l'intégration de repères culturels et historiques, référents pour la discipline est loin d'être propre à l'enseignement de la littérature. La prise de conscience par l'apprenant de ses représentations initiales, parfois stéréotypées, est largement valorisée et pratiquée en sciences. Dès lors, on peut émettre les deux hypothèses suivantes: d'une part, travailler la distanciation au stéréotype dans le cadre de l'écriture littéraire contribue également au développement plus global de la capacité fondamentale à prendre distance; d'autre part, l'entrée par le matériau stéréotypie ou stéréotype demeure pertinente pour travailler la distanciation 
quel que soit le champ considéré, dans la mesure où ce matériau intervient dans le dire, le faire et le penser de toute discipline et que, pour qu'il y ait apprentissage, il est souvent nécessaire de le mettre à distance.

\section{Prendre distance et multiplier les indices et les opérations: un effort minimal?}

Les opérations pointées ci-dessus sont-elles facilement réalisables par des élèves de fin d'école primaire? À observer combien le stéréotype s'avère être un phénomène résistant par rapport auquel certains élèves ont du mal à prendre distance, il apparaît que la difficulté d'abstraction, de relativisation, de multiplication, de prise de conscience, d'interprétation "imaginante ", d'ouverture à l'étrange, de catégorisation plus générale et de resserrement du rôle ou de la zone de pertinence n'est vraiment pas à minimiser. Le risque d'une sous-évaluation de ces opérations et de leur difficulté serait, selon nous, de passer à côté d'une réelle transformation, d'une réelle mise en mouvement du figement de la représentation et de ses manifestations langagières.

La difficulté est notamment due au fait que le stéréotype a tendance à avancer masqué et à harmoniser ou homogénéiser l'activité (Kervyn, 2008 b et 2008 d). Ainsi facilitée, celle-ci ne suscite pas le questionnement et la problématisation du stéréotype. C'est particulièrement visible dans la classe 2 , où l'usage de "nouveaux » stéréotypes pour adopter un autre point de vue sur la mer facilite la réécriture. Ainsi, des vacances idylliques à la mer, on passe par exemple à une vision d'une mer monstrueuse à laquelle est associé tout l'attirail du danger et de la catastrophe. Si cet usage spontané du stéréotype forme un outil équitable dans la mesure où il facilite l'écriture du plus grand nombre, il ne pousse pas les élèves à un nouvel usage distancié. On est ainsi en présence d'un phénomène qui a tendance à anesthésier la conscience. En conséquence, il ne faut pas minimiser la difficulté que peut représenter, pour l'enseignant mais surtout pour les élèves, l'opération de prise de conscience et de distance du phénomène. Cela étant, cette évolution scripturale montre bien combien le stéréotype peut outiller l'écriture, même s'il ne paraît pas être utilisé consciemment. L'outillage par le stéréotype est alors double: secondarisation de l'écriture par mise à distance du premier stéréotype (association de la mer aux vacances idylliques) et prise de distance par adoption du second stéréotype (association de la mer à la peur et au danger). Apparaît du même coup l'imbrication fonctionnelle possible entre les postures participative et distanciée.

\section{Voir la diversité scripturale et la renforcer}

81 La prise en compte des données recueillies auprès des élèves met en avant une importante diversité initiale et finale en matière de traitement écrit du stéréotype. $\mathrm{Si}$, dans la classe 1 par exemple, la représentation de la poésie en tant que « genre " rimé bloque l'écriture pour certains élèves, elle la facilite chez d'autres, pendant que certains élèves encore l'ont déjà relativisée. Dans les deux classes, cette diversité a pourtant tendance à être omise par les enseignants qui en font une lecture homogénéisante Kervyn, 2008 b). Le risque est alors de passer à côté d'une richesse textuelle et du niveau réel de chaque élève concernant le traitement des stéréotypes. Dans ce sens, une des conditions pour faire du stéréotype un outil d'enseignement et d'apprentissage de l'écriture serait de porter attention aux hétérogénéités discrètes que masque le fonctionnement homogénéisant du stéréotype. 
82 Par ailleurs, l'attention à la diversité scripturale mise en œuvre par les élèves nous amène à relativiser une conception linéaire de l'enseignement des stéréotypes, laquelle consisterait à enseigner successivement la maîtrise d'un stéréotype, la participation à celui-ci, puis seulement sa mise à distance pour ensuite susciter l'appropriation d'un nouveau stéréotype un peu plus élaboré et ainsi de suite. Une analyse discursive des textes d'élèves indique des usages multiples, des figements et défigements divers, quel que soit le stéréotype concerné. Elle nous incite dès lors à ne pas concevoir l'apprentissage de l'écriture littéraire seulement comme la maitrise successive de stéréotypes de plus en plus complexes, mais aussi comme la mise en œuvre de traitements textuels divers des stéréotypes (renversement, alternance, interférence, allusions négatives, etc.) ${ }^{38}$ et de mouvements de fictionnalisation marqués par des rapports multiples entre le vécu du scripteur et l'imaginaire collectif stéréotypé plus ou moins incorporé (Kervyn, 2009 a).

\section{Outillage et innovation}

Parler d'innovation en ayant pour outil la stéréotypie peut sembler contradictoire. Selon nous, pourtant, l'innovation, qui se caractérise notamment par la transformation des pratiques (Cros, 2004), est à mettre en lien avec l'outillage didactique professionnel des enseignants. En effet, nous constatons que l'outil utilisé, en l'occurrence la notion de stéréotype, amène les enseignants collaborateurs à mettre en place de nouvelles pratiques. L'innovation, bel et bien présente, est générée, en partie au moins, par l'outil et par des conditions favorisant sa réalisation, en l'occurrence ici le protocole d'expérimentation retenu. En laissant une grande liberté aux enseignants collaborateurs, en respectant leur profil d'implication et de collaboration, ce protocole fait d'eux les principaux acteurs de leur développement professionnel, tel que le conçoit Gather Thurler (2004), et il permet le recueil de données multiples et variées.

On voit dès lors l'importance pour la didactique de dégager des conditions ou des modalités de réalisation de l'outillage, qu'elles soient de nature méthodologique ou qu'elles concernent la place à octroyer à la conceptualisation, à la réflexion pour apprendre à écrire, ou encore des gestes professionnels ponctuels ${ }^{39}$. Dans tous les cas, ces modalités ou ces conditions nous paraissent avoir permis, outre un enrichissement des savoirs didactiques, un développement professionnel des enseignants et/ou un développement de la capacité d'écrire de la poésie chez les élèves, et cela même si ces formes de développement dépassent nos observations, qui étaient centrées sur la mise en œuvre scolaire de l'enseignement et de l'apprentissage.

\section{Conclusion}

Pour clore cet article par une mise en dialogue des deux approches de la stéréotypie que nous avons proposées, nous voudrions aborder la question suivante: quelles questions pose l'analyse de pratiques au modèle que nous avons posé dans notre première partie? En quoi l'opérationnalisation réalisée renforce-t-elle, interroge-t-elle ou enrichit-elle un modèle descriptif général, qui, forcément, qui n'était pas centré a priori sur le contexte d'enseignement et d'apprentissage de l'écriture à l'école primaire? 
Dans une logique de croisement des deux approches, cinq points retiennent notre attention.

1. Tout d'abord, pour ce qui est de l'outillage des enseignants puis des élèves, l'expérimentation attire notre attention sur le fait que la construction de la signification et de la valeur didactique fonctionnelle du stéréotype s'effectue difficilement sans influence du sens et de la valeur négative couramment attribués au stéréotype. Les tensions relevées rappellent que, dès lors que les acteurs connaissent et partagent ce sens courant, ils n'y échappent pas aisément, même si le travail didactique mené s'inscrit dans le sillage de modélisations théoriques qui s'en écartent.

2. Notre second point concerne les élèves: qu'est-ce qu'«assumer » un stéréotype pour des élèves de 10-11 ans qui développent leurs capacités langagières depuis leur plus jeune âge dans et par les mots des autres? C'est avant tout l'avoir intériorisé et le véhiculer, le plus souvent comme une pensée et une parole singulière ou reprise à un discours ou à une situation précise. « Assumer » le stéréotype, ce n'est donc pas majoritairement y adhérer en tant que stéréotype, ni y recourir en connaissant certains de ses traits caractéristiques (notamment sa récurrence ou son usage partagé par une communauté). Nous parlerions plus volontiers de reprise, de réutilisation d'un phénomène qui relève du dialogisme constitutif (Authier Revuz, 1982). Bien que le fonctionnement dialogique de la stéréotypie soit fortement accentué (Boré, 2007) vu qu'on a affaire à des reprises fréquentes, durables et effectuées par un grand nombre d'individus, cette accentuation n'est pas d'emblée perçue par les élèves scripteurs, qui y voient plutôt un usage ou une reprise singulière. Plus généralement, ce constat renvoie au filtre culturel de la perception et à l'intrication du social et de l'individuel, du générique et du spécifique.

87 À ce propos, on peut penser qu'en situation, aussitôt que l'activité est facilitée ou homogénéisée par l'usage du stéréotype, elle ne favorise pas le questionnement du phénomène. Dès lors, si dans ce cas le stéréotype apporte bien une aide importante à l'écriture, celle-ci tient à l'usage d'un matériau fréquent, stable et partagé, mais non à la prise de conscience de ces traits. Nous avons vu en effet que la prise de conscience et la conceptualisation ne sont pas indispensables pour qu'il y ait outillage du scripteur, et qu'elles n'empêchent pas le phénomène d'agir de façon masquée en situation d'écriture. Elles permettent néanmoins de constater un usage partagé et de comprendre pourquoi on a régulièrement tendance à y revenir, à s'exprimer et à penser de cette manière.

1. Est-il possible de distinguer dans le processus scriptural observé le deuxième et le troisième degré de traitement des stéréotypes? En l'occurrence, ne pourrait-on pas rapprocher la progression scripturale des élèves de l'adoption d'une posture du troisième degré, qui combine l'adhésion et la mise à distance? À vrai dire, il est difficile de se prononcer. Tout ce qu'on peut constater, c'est que l'évolution dont témoignent les écrits des élèves tient à de multiples mises en mouvement des stéréotypes initiaux, qu'ils soient thématiques ou formels, et que ces mises en mouvement apparaissent comme fortement liées aux opérations de distanciation relevées dans les classes. C'est pourquoi, d'un point de vue didactique, il semble d'abord opératoire de mettre l'accent sur ce processus de distanciation.

88 Cela étant, le fait que la posture du $3^{\mathrm{e}}$ degré soit peu aisée à distinguer de celle du $2^{\mathrm{e}}$ dans les écrits des élèves ne signifie nullement qu'il s'agisse d'une posture esthétique réservée aux élites et n'empêche nullement de la maintenir comme un objectif pédagogique important. Winnicott (1971), parmi d'autres, a bien montré que l'espace transitionnel du va-et-vient était une expérience fondamentale pour le développement psychologique et cognitif de tous les individus. Rien n'est donc plus « ordinaire » que de combiner la participation et la distanciation, mais cette attitude de va-et-vient qui 
permet de construire la bivalence fondamentale des stéréotypes et leur valeur fonctionnelle requiert un apprentissage scolaire dans diverses disciplines.

1. Peut-on traiter de façon distincte, à propos de nos données de terrain, les quatre niveaux de réalité concernés par la stéréotypie? Si leur distinction est nécessaire pour saisir le fonctionnement et l'étendue des phénomènes de stéréotypie, dans les écrits des élèves, on relève surtout leur forte imbrication. De fait, les comportements langagiers nous sont apparus à la fois inscrits dans une dimension générique et marqués par des représentations ou des idées renvoyant à des marques de figement verbal. Si l'on admet que, dans l'écriture au moins, les représentations mentales stéréotypées (qui correspondent au niveau de l'inventio) ne peuvent être isolées de leurs manifestations langagières et textuelles, opérationnaliser des phénomènes de stéréotypie pour travailler l'écriture exige de se situer à l'articulation du cognitif, du discursif et du textuel. En d'autres mots, s'agissant des représentations stéréotypées, plutôt que de chercher à les étudier sur un plan strictement cognitif, il s'agit de les envisager en discours et au « ras du texte », dans le souci de la langue (Fenoglio, 2007).

2. Par ailleurs, le travail sur les représentations stéréotypées a pris place et forme dans l'étude d'un objet-clé en didactique de l'écrit, à savoir le genre, ici envisagé via sa structure formelle et son contenu thématique. On peut légitimement penser que, selon les contenus disciplinaires, le travail se découpera diversement selon les objets mais nécessitera toujours un redéploiement au plus près des unités considérées ${ }^{40}$. Car c'est bien au cœur de cette complexité que se situent l'outillage et l'étude didactique de la stéréotypie.

Ainsi, même si notre étude n'a pris appui que sur deux classes de français, dans la mesure où les gestes professionnels des enseignants semblent, pour une large part, relever de schèmes et véhiculer des valeurs apparemment récurrentes dans la communauté des enseignants (comme celles de la création et de l'originalité), on est en droit d'espérer que les contraintes et les potentialités que fait ressortir un tel travail pourront outiller d'autres chercheurs, d'autres enseignants, et ainsi faire progresser quelque peu les fondements épistémologiques, praxéologiques et méthodologiques, non seulement de la discipline «français ", mais aussi d'autres didactiques, voire, en dernier recours, le concept même de didactique.

\section{BIBLIOGRAPHIE}

Alcorta, M. (1998).Une approche vygotskienne du développement des capacités d'écrit. Le brouillon: un outil pour écrire. In Brossard, M. \& Fijalkow, J. Apprendre à l'école: perspectives piagétiennes et vygotskiennes. Bordeaux: Presses Universitaires de Bordeaux, 123-151.

Alcorta M. (2009).Le mythe de l'égalité des chances. In Actes du colloque international Efficacité et équité en éducation. Rennes (à paraître).

Amossy, R. (1991). Les idées reçues. Sémiologie du stéréotype. Paris: Nathan.

Amossy, R. et Herschberg Pierrot, A. (1997). Stéréotypes et clichés. Langue, discours, société. Paris: Nathan (128). 
Authier-Revuz, J. (1982). Hétérogénéité montrée et hétérogénéité constitutive: éléments pour une approche de l'autre dans le discours. In DRLAV, 17, 1-78.

Bakhtine, M. (1984).Esthétique de la création verbale. Paris: Gallimard.

Barbier, J.-M. (dir.) (1996). Savoirs théoriques et savoirs d'action. Paris: PUF.

Bautier, É. \& Goigoux, R. (2004). Difficultés d'apprentissage, processus de secondarisation et pratiques enseignantes: une hypothèse relationnelle. Revue Française de Pédagogie, 148, 89-100.

Bernié, J.-P. (dir.) (2002). Apprentissage, développement et significations. Hommages à Michel Brossard. Bordeaux: Presses Universitaires de Bordeaux.

Bernié, J.-P. (2002), L'approche des pratiques langagières scolaires à travers la notion de « communauté discursive » : un apport à la didactique comparée? Revue Française de Pédagogie, $141,77-88$.

Boré, C. (2007). L'écriture scolaire entre stéréotype et idiolecte. Pratiques, 135-136,217-239.

Boyer, H. (2007) (dir.). Stéréotypage, stéréotypes: fonctionnements ordinaires et mises en scène. Paris: L'Harmattan.

Bronckart, J.-P. \& Schneuwly, B. (1985). Vygotsky aujourd'hui. Neuchâtel - Paris: Delachaux et Niestlé.

Brossard, M. (2004). Vygotski. Lectures et perspectives de recherches en éducation. Villeneuve d'Ascq: Presses Universitaires du Septentrion.

Brossard, M. (1998). Approche socio-historique des situations d'apprentissage de l'écrit. In Brossard, M. \& Fijalkow, J. Apprendre à l'école: perspectives piagétiennes et vygotskiennes. Bordeaux: Presses Universitaires de Bordeaux, 37-50.

Brossard, M. (1989). Espace discursif et activités cognitives. Enfance, 42, 49-56.

Bruner, J. (1983, rééd. 2002). Le développement de l'enfant: savoir faire, savoir dire. Paris: PUF.

Ceysson, P. (2004). Faire écrire de la poésie: six formules plus une pour les ateliers d'écriture poétique. L'École des lettres des collèges, 13, 135-148.

Chevallard, Y. (1994). Les processus de transposition didactique et leur théorisation. In Arsac, G., Chevallard, Y., Martinand J.-L. \& Tiberghien, A. La transposition didactique à l'épreuve. Grenoble: La Pensée Sauvage, 135-180.

Chiss, J.-L. (1993). Hétérogénéité des apprenants et traitements des contenus disciplinaires. In Lebrun, M. \& Paret, M.-C. L'hétérogénéité des apprenants. Un défi pour la classe de français, Neuchâtel - Paris: Delachaux et Niestlé, 180-183.

Clot, Y. (1999/2004). La fonction psychologique du travail. Paris: PUF.

Cros, F. (2004). L'innovation scolaire aux risques de son évaluation. Paris: L'Harmattan.

Daunay, B. (2002). Le lecteur distant. Positions du scripteur dans l'écriture du commentaire. Pratiques, 113-114, 135-153.

Diptyque (2006). $\mathrm{n}^{\circ}$ 5. Didactique de l'écrit. La construction des savoirs et le sujet-écrivant.

Dreyfus, M. (2007). Production et déplacement de stéréotypes en situation d'entretien. In Boyer, H. (dir.). Stéréotypage, stéréotypes: fonctionnements ordinaires et mises en scène (4). Paris: L'Harmattan, 77-87.

Dufays, J.-L. (1991). Lire avec les stéréotypes. Les conditions de la lecture littéraire en classe de français. Enjeux, 23, Aimer lire, Namur, 5-18. 
Dufays, J.-L. (1993). Initier à la conscience des stéréotypes: un enjeu éthique essentiel du cours de français. Enjeux, 29, Namur, 47-59.

Dufays, J.-L. (1994). Stéréotype et lecture. Essai sur la réception littéraire. Liège: Mardaga (Philosophie et langage), $375 \mathrm{p}$.

Dufays, J.-L. (1997). Stéréotypes et didactique du français. Histoire et état d'une problématique. Études de linguistique appliquée, 107, 315-328.

Dufays, J.-L. (2001). Le stéréotype, un concept clé pour lire, penser et enseigner la littérature. Marges linguistiques (revue web) et in Gauthier, R. (dir.), Le stéréotype: usages, formes et stratégies. Actes du $21^{e}$ colloque d'Albi Langages et signification, Toulouse, C.A.L.S./CPST, 2001, 19-30.

Dufays, J.-L. \& Vanhulle, S. (2002). La lecture dans tous ses états. Quelques pistes pour (bien) l'enseigner. Enjeux, 55, Former des enseignants de lecture, 13-30.

Dufays, J.-L. (2006). Stéréotypes, apprentissage, interculturalité: fondements théoriques et pistes didactiques. In L. Collès, J.-L. Dufays \& Fr. Thyrion(dir.).L'interculturel en francophonie. (1). Problématique générale, Cortil-Noirmont, E.M.E. (Didactiques), 57-84.

Dufays, J.-L. (2007). Stéréotyper, suspendre, rouvrir: le chantier sans fin de la lecture et de l'apprentissage. In Boyer, H. (dir.). Stéréotypage, stéréotypes: fonctionnements ordinaires et mises en scène (3). Paris: L’Harmattan, 81-89.

Eco, U. (1985a). Lector in fabula ou la coopération interprétative dans les textes narratifs. Paris: Grasset (Figures) (éd. orig. : 1979).

Eco, U. (1985b). Apostille au Nom de la Rose. Paris: Grasset (Le Livre de Poche Biblio Essais).

Études de linguistique appliquée (1997). 107, Stéréotypes et alentours.

Fenoglio, I. (dir.) (2007). L'écriture et le souci de la langue. Écrivains, linguistes: témoignages et traces manuscrites. Louvain-la-Neuve: Académia-Bruylant.

François, F. (2006). Rêves, récits de rêves et autres textes. Un essai sur la lecture comme expérience indirecte. Limoges: Éditions Lambert-Lucas.

François, F. (2005a). Subjectivation, constances et variations dans quelques textes d'élèves de CM2. Langage et société, 111, 73-95.

François, F. (2005b). Interprétation et dialogue chez des enfants et quelques autres. Lyon: ENS Éditions. Galatanu, O. (2007). Pour une approche sémantico-discursive du stéréotypage à l'interface de la sémantique théorique et de l'analyse du discours. In Boyer, H. (dir.). Stéréotypage, stéréotypes: fonctionnements ordinaires et mises en scène (4). Paris: L'Harmattan, 89-100.

Gather Thurler, M. (2004). Stratégies d'innovation et place des acteurs. In Bronckart, J.-P. et Gather Thurler, M. Transformer l'école. Bruxelles: De Boeck, 99-125.

Le Français aujourd'hui (1996), 113, Interactions: dialoguer, communiquer.

Iser, W. (1985). L'acte de lecture. Théorie de l'effet esthétique. Liège: Mardaga (Philosophie et langage) (éd. orig.: 1976).

Jaubert, M. (2007). Langage et construction de connaissances à l'école. Un exemple en sciences, Bordeaux: Presses Universitaires de Bordeaux.

Jaubert, M. et Rebière, M. (2005). Emergence d'un concept en didactique du français: la secondarisation. Actes du colloque international Didactiques: quelles références épistémologiques? cédérom, IUFM d'Aquitaine - AFIRSE. 
Kervyn, B. (2009 a).Écriture poétique et fictionnalisation en classe de CM2. In Plane, S. et Dufays, J.-L. (dir.). L'écriture de fiction en classe de français. Laval: Presses Universitaires de Laval, 149-166.

Kervyn, B. (2009 b). Dépliage du processus de distanciation pour écrire de la littérature en fin d'école primaire. Repères, 40, 155-174.

Kervyn, B. (2008 a). Didactique de l'écriture et phénomènes de stéréotypie. Le stéréotype comme outil d'enseignement et d'apprentissage de l'écriture poétique en fin d'école primaire. Thèse de doctorat. Louvain-la-Neuve: Université Catholique de Louvain, $1150 \mathrm{p}$.

Kervyn, B. (2008 b).Écriture scolaire et stéréotypie. Processus d'homogénéisation et mises en œuvre hétérogènes. Repères, 38, 167-185.

Kervyn, B. (2008 c). Étude et opérationnalisation de phénomènes de stéréotypie pour la didactique de l'écriture. In Loiseau, M., Abouzaid, M., Buson, L. (dir.). Autour des langues et du langage: perspective pluridisciplinaire. Grenoble: Presses Universitaires de Grenoble, 389-396.

Kervyn, B. (2008 d). Le socle commun et l'écriture dans le socle commun: observations sous l'angle de la stéréotypie. In Dubois-Marcoin D. \& Tauveron C. (dir.). Français, langue et littérature, socle commun: quelle culture pour les élèves, quelle professionnalité pour les enseignants? . Lyon: INRP, 167-178.

Kervyn, B. (2007). Les phénomènes de stéréotypie: essai d'outillage pour la didactique de l'écriture. In Boyer, H. (dir.). Stéréotypage, stéréotypes: fonctionnements ordinaires et mises en scène (3). Paris: l'Harmattan, 151-162.

Kervyn, B. (2006). Une formation au service de la recherche et une recherche au service de la formation, in Robert, A. (dir.). Formation des enseignants: quels scénarios? Quelles évaluations? Actes du colloque IUFM d'Anthony Val de Bièvre, cédérom, mars2006.

Kervyn, B. \& Faux, J. (2007). Écriture poétique et stéréotypie en classe de CM2: comment les différents acteurs impliqués donnent-ils sens et valeurs au travail réalisé?. In Dufays, J.-L. (dir.). Enseigner et apprendre la littérature aujourd'hui, pour quoi faire? Sens, utilité, évaluation. Louvain-laNeuve: Presses Universitaires de Louvain, 243-254.

Kleiber, G. (1990/2004). La sémantique du prototype. Paris: Presses Universitaires de France.

Ministère de l'Éducation nationale (2002). Qu'apprend-on à l'école élémentaire? Paris: Scéren CNDP / XO Éditions.

Paquay, L., Crahay, M. \& De Ketele, J.-M. (2006). L'analyse qualitative en éducation. Bruxelles: De Boeck.

Pépin, N. (2007). Stéréotypes en interaction. Éléments d'une grammaire de l'identité. In Boyer, H. Stéréotypage, stéréotypes: fonctionnements ordinaires et mises en scène. Paris: L'Harmattan, 191-202.

Petitjean, A. (1998). Enseignement/apprentissage de l'écriture et transposition didactique.

Pratiques, 97-98, 105-132.

Picard, M. (1986). La lecture comme jeu, Paris: Minuit (Critique).

Rabardel, P. (1999/2002). Le langage comme instrument? Éléments pour une théorie instrumentale étendue. In Clot, Y. (dir.). Avec Vygotski, Paris: La Dispute, 265-290.

Rabardel, P. (1997). Activités avec instruments et dynamique cognitive du sujet.In Moro, C. et Schneuwly, B. Outils et signes. Perspective actuelle de la théorie de Vygotski. Neuchâtel: Peter Lang. Recherches (1989), Stéréotypes et apprentissages. 
Schneuwly, B. (1985).La construction sociale du langage écrit chez l'enfant. In Bronckart, J.-P. \& Schneuwly, B. (dir.). Vygotsky aujourd'hui. Neuchâtel - Paris: Delachaux et Niestlé, 169-202.

Schneuwly, B. Dolz, J. et Ronveaux, C. (2006). Le synopsis: un outil pour analyser les objets enseignés. In Perrin-Glorian, M.-J. et Reuter, Y. Les méthodes de recherche en didactiques. Lille: Presses Universitaires du Septentrion, 175-190.

Siméon, J.-P. (2003). Le problème avec la poésie. Cahiers pédagogiques, ${ }^{\circ}$ 417, 9-11.

Stevenson, C.L. (1992). Qu'est-ce qu'un poème? In Genette, G. Esthétique et poétique. Paris: Seuil, 157-202.

Tauveron, C. (1999). Comprendre et interpréter le littéraire à l'école: du texte réticent au texte proliférant. Repères, 19, 21-50.

Van Dijk, T.A. (1977). Text and Context. New York: Longman.

Vergnaud, G. (2000). Lev Vygotski. Pédagogue et penseur de notre temps. Paris: Hachette Éducation.

Vincent, C., Delozanne, E., Grugeon, B., Gélis, J.-M., Rogalski, J., Coulange, L. (2005). Des erreurs aux stéréotypes: des modèles cognitifs de différents niveaux dans le projet Pépite. Scientific Commons, publication en ligne http://hal.archives-ouvertes.fr/hal-00005689/en/.

Vygotski, L.S. (1934/1985/1997). Pensée et langage. Paris: La Dispute.

Winnicott, D. W. (1971). Jeu et réalité. L'espace potentiel. Paris : Gallimard.

Wirthner, M. (2006). La transformation de pratiques d'enseignement par l'outil de travail. Observation de séquences d'enseignement du résumé écrit de texte informatif à l'école secondaire. Thèse de doctorat en Sciences de l'éducation. Genève: Université de Genève.

\section{NOTES}

1. Approche qui intègre aussi ce que nous appelons les " phénomènes de stéréotypie ».

2. Une précision toutefois: notre description vise ici les lecteurs qui ont déjà appris à lire. Un certain nombre de nuances, que nous ne ferons pas ici car ce n'est pas notre propos, seraient nécessaires pour décrire le cas des enfants qui apprennent à lire.

3. Entendons-nous bien: l'image verbale, le mot ou la signification ne sont pas en soi «des stéréotypes ", mais le lecteur qui associe des signifiants, des signifiés et des référents de manière automatisée stéréotypise dans la mesure où il effectue des associations qui répondent au critère précédent.

4. Pour cette distinction entre interprétation de niveau 1 et de niveau 2, voir Tauveron, 1999.

5. Cinquième année primaire en Belgique.

6. Outre les deux enseignants collaborateurs et la chercheuse, le groupe de travail comptait 5 professeurs des écoles aussi maîtres formateurs, tous intéressés par le projet et diversement impliqués.

7. Voir Kervyn, 2006.

8. Il s'agit de Thierry Lamarque et de Jérôme Faux, dont nous citons les noms, avec leur accord, en guise de reconnaissance pour le travail réalisé et l'intérêt porté à cette recherche.

9. Pour limiter le flou ou les malentendus méthodologiques et épistémologiques qui, parfois, découlent de la disparité des conceptions et du foisonnement des pratiques qui se réclament de la recherche-action, nous avons tenté de redéfinir ce type de démarche (Kervyn, 2008a) dans le paysage actuel de la recherche, caractérisé notamment par un indispensable souci de scientificité ou par la nécessité de critères de qualité (Paquay, Crahay et De Ketele, 2006). 
10. Dans le sens bakhtinien, où tout énoncé est traversé par la présence du discours d'autrui (Bakhtine, 1994).

11. Le sens ici attribué à "secondarisé" ou à "secondarisation" recoupe les approches sociologiques et didactiques qu'en font Bautier et Goigoux (2004) ainsi que Jaubert et Rebière (2005) : chacun à leur façon, ces auteurs prônent une secondarisation des savoirs et des pratiques scolaires à travers un mode d'apprentissage cognitivo-verbal qui relève de la distanciation.

12. Dont Ceysson (2004), Siméon (2003), Stevenson (1992).

13. Dans les traductions françaises de l'œuvre de Vygotski comme dans les commentaires faits sur son œuvre, les termes « outil » et « instrument » sont tous deux utilisés et semblent le plus souvent équivalents. Par contre, dans les travaux de Rabardel (1997, 1999/2002), qui poursuit la réflexion débutée par Vygotski sur les instruments psychologiques, on trouve un emploi différencié de ces deux termes: l'outil désigne d'abord un artefact (objet fabriqué) et ne devient instrument que « [...] lorsqu'il est associé à l'activité des utilisateurs de l'outil » (Vergnaud 2000, p. 85), renvoyant à l'article de Rabardel publié dans Avec Vygotski (1999/2002). En parallèle, les équipes genevoise et bordelaise privilégient, semble-t-il, le terme outil (Alcorta, 1998; Brossard, 2004; Jaubert, 2007; Schneuwly, 1985; Wirthner, 2006...). Pour notre part, malgré l'intérêt que nous portons aux travaux de Rabardel, nous continuerons à privilégier le terme outil.

14. On peut y voir une forme de mémorisation, mais qui prend appui sur de l'interaction et de la reconfiguration (François, 2005 a et b).

15. On peut faire allusion au passage du mot stéréotype qui identifie (fonction d'identification) au même mot qui conceptualise (fonction de conceptualisation).

16. Nous n'utilisons pas ici le terme activité dans son acception générale, renvoyant à l'agir humain. Tel qu'employé, activité désigne une unité de travail scolaire située au point de rencontre entre la tâche demandée par l'enseignant et la tâche remodelée par les élèves qui la réalisent (Schneuwly, Dolz \& Ronveaux, 2006).

17. C'est-à-dire que la pratique scolaire considérée ne se réduit pas à la planification effective du travail par l'enseignant; elle intègre aussi les ajustements réalisés dans l'interaction avec les élèves.

18. Le processus d'opérationnalisation s'effectue en classe et hors de la classe, in vivo et in bureau, en direct et indirectement, dans la mesure où le questionnement et l'analyse des données recueillies en classe font aussi partie intégrante du processus d'opérationnalisation. Ils éclairent et construisent le processus d'outillage.

19. Pour consulter les données ainsi que leur présentation détaillée et leur analyse, voir Kervyn, 2008 a, volume1 pour la présentation et l'analyse, volume2 pour la consultation des données.

20. Comme l'enseignant le précise (Kervyn \& Faux, 2007, p. 247), cette démarche de travail repose elle-même sur des valeurs idéologiques et pédagogiques, à savoir sur la conviction que tous les élèves sont capables de réfléchir sur leur façon de réfléchir et d'écrire, y compris ceux qui n'ont pas une forte culture de l'écrit. On pourrait parler ici d'une conception égalitaire et d'une croyance en la capacité des élèves à entrer dans les savoirs et à y entrer d'autant plus profondément s'ils sont co-acteurs de leur construction.

21. La distinction entre les genres littéraires et les genres scolaires en littérature est fondamentale pour la poésie. En effet, dans la communauté littéraire, l'étiquetage de la poésie en tant que genre est souvent contesté, vu la diversité des formes existantes (sonnet, calligramme, haïku, poème en prose, etc.), qui peuvent chacune former un genre particulier. À l'école primaire, la poésie est bien abordée en tant que genre, au même titre que le conte, le roman, le théâtre, la nouvelle. Il s'agit alors d'un redécoupage institutionnel, d'un classement, par le milieu et pour le milieu, d'objets existant par ailleurs. Dans une perspective bakhtinienne, on peut considérer qu'il s'agit d'une forme discursive stabilisée et partagée au sein de la communauté discursive scolaire. 
22. Nous sommes conscients de notre part d'inférence dans la description de ces objectifs et dans l'attribution des activités à tel ou tel objectif, car nous reconstruisons des logiques d'actions enseignantes qui n'ont pas toujours été explicitées (voire perçues de manière consciente) ou qui ne l'ont pas toujours été dans ces mots.

23. Soit les stéréotypes, les prototypes, les clichés, les lieux communs, les idées reçues, les locutions ou expressions figées, les topoï, la stéréotypisation (ou le stéréotypage).

24. L'autre enseignant a lui aussi donné ultérieurement au stéréotype une dimension transversale en le réutilisant comme outil en sciences, dans un travail sur les phases de la lune comportant des écritures successives en vue de rendre compte de l'expérimentation et d'outiller le processus d'expérimentation et de construction des savoirs (Kervyn, $2008 \mathrm{c}$ ).

25. L'abréviation $\mathrm{E}$ désigne un élève.

26. § §§ marquent, à l'intérieur d'un énoncé, le début et la fin d'un chevauchement de paroles.

27. Cela laisse en suspens la question de savoir si leur présence est due au fait que l'activité scripturale demandée est complexe ou si elle est indépendante de la complexité de l'activité demandée.

28. Bakhtine utilise les termes de construction compositionnelle (1984, p. 265).

29. À laquelle s'ajoute le style.

30. Ces deux objectifs sont d'ailleurs bien en accord avec les programmes de 2002 alors en vigueur, selon lesquels « [l'] univers de [la] littérature se découvre aussi, dès l'école primaire, par la pratique de l'écriture. Cette expérience, plus exigeante [que celle de la lecture], permet à l'élève de commencer à prendre conscience des spécificités du monde des fictions » (MEN, $2002 \mathrm{a}$, p. 233-234).

31. Un article, publié dans le numéro40 de la revue Repères, est consacré à ce sujet (Kervyn, 2009b).

32. Cette deuxième opération, dans les observations réalisées, apparaît comme fortement liée à la première. En effet, d'une part, on est en présence d'une connaissance, d'une activation ou d'une valorisation d'indices de poéticité restreints peu relativisés. D'autre part, relativiser l'importance ou l'aspect incontournable d'un stéréotype peut aussi bien être une condition pour s'ouvrir à d'autres points de vue que le résultat de cette ouverture.

33. «Nouveaux » pour les sujets qui les énoncent ou nouveaux par rapport à leurs discours antérieurs.

34. Au lieu de déplacer le regard vers de multiples « nouveaux » indices ou points de vue, comme dans l'opération précédente, le mode opératoire consiste ici à en privilégier un seul. En écriture, distinguer ces deux formes de distanciation importe car, contrairement au temps d'échange oral sur l'écriture ou sur la poésie, on constate globalement une tendance au resserrement des points de vue ou des indices sollicités.

35. Seul ce processus n'a pas été observé mais a semblé pertinent à susciter au vu des besoins constatés.

36. Sans cet écart, le stéréotype place en terrain connu, active des réseaux sémantiques balisés car stabilisés et récurrents. Il ne surprend pas et même entrave l'imagination et la diversité générique.

37. Ce choix n'est pas à minimiser si l'on postule, dans la lignée de Vygotski (1934/1985), de Bruner (1983) ou de Rabardel (2002), que la conscience est outil. Prendre conscience du fait que la représentation selon laquelle un poème a toujours des rimes est un stéréotype, modifie le rapport du sujet au poème, à la rime et à leur lien. Les savoirs sur les phénomènes de stéréotypie auraient pu servir uniquement à l'enseignant pour diagnostiquer la présence de stéréotypes et ensuite travailler sur leur assouplissement par une multiplication des indices, sans s'employer à faire prendre conscience aux élèves du processus de stéréotypisation à l'œuvre. Dans un cas, les savoirs sur la stéréotypie ne sont directement outil que pour le maître, dans l'autre ils outillent aussi directement les élèves. 
38. Pour un détail de ces divers traitements textuels exemplifiés par les textes des élèves, voir Kervyn, 2008a.

39. Ces gestes, tel le recours au dictionnaire pour découvrir le sens du terme stéréotype, peuvent, nous l'avons évoqué, être lourds de conséquence.

40. Voir notamment les travaux sur les stéréotypes menés par C. Vincent et al. (2005) dans le domaine des mathématiques.

\section{RÉSUMÉS}

Deux approches de la stéréotypie sont mises en dialogue afin d'affiner sa constitution en objet didactique. La première, de type modélisant, distingueles différents niveaux de stéréotypie envisageables et analyse leurs manifestations dans les processus de lecture et d'écriture. Les traitements du premier degré, qui utilisent les stéréotypes comme des signes ordinaires, sont ainsi opposés à ceux du deuxième degré, qui en font des citations perçues comme telles, et à ceux du troisième degré, qui mettent les deux premiers en tension. Dans la seconde approche, de type expérimental, on observe la manière dont le stéréotype est mis en œuvre comme outil au service de la didactique de l'écriture littéraire par des enseignants de l'école primaire et les traitements dont il est l'objet de la part des élèves. La confrontation de ces deux approches permet de se demander dans quelle mesure l'opérationnalisation réalisée renforce, interroge ou enrichit le modèle descriptif initial.

Two approaches of stereotypy are put in dialogue in order to refine its establishment as didactic object. The first one, of modelling type, distinguishes between the various levels of stereotypy and analyzes their appearances in the reading and writing processes. The treatments of the first degree, which use stereotypes as common signs, contrast then with those of the second degree, which turn them into quotations being perceived as such, and with those of the third degree, which put the first two treatments in tension. In the second approach, of experimental type, we observe how primary school teachers implement the stereotype as a tool in the service of literary writing didactics and how pupils deal with it. The confrontation of these two approaches allows us to wonder to what extent the experiment that was carried out strengthens, questions or enriches the initial descriptive model.

\section{INDEX}

Mots-clés : écriture, lecture, littérature, outillage didactique, Stéréotypie

Keywords : didactic tool, literature, reading, Stereotypy, writing

\section{AUTEURS}

JEAN-LOUIS DUFAYS

UCL, CRIPEDIS / CEDILL 


\section{BERNADETTE KERVYN}

IUFM d'Aquitaine - Université Bordeaux 4, LACES-DAESL 